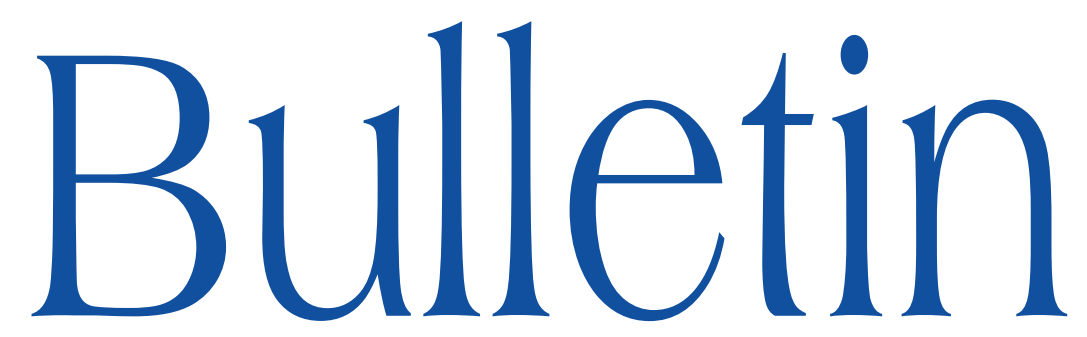

de la SOCIÉTÉ MATHÉMATIQUE DE FRANCE

\title{
SUR LA PERSISTANCE DES COURBES INVARIANTES POUR LES DYNAMIQUES HOLOMORPHES FIBRÉES LISSES
}

Mario Ponce

Tome 138

Fascicule 2

2010 


\title{
SUR LA PERSISTANCE DES COURBES INVARIANTES POUR LES DYNAMIQUES HOLOMORPHES FIBRÉES LISSES
}

\author{
PAR MARIO PONCE
}

\begin{abstract}
RÉsumé. - En s'appuyant sur un théorème des fonctions implicites de Hamilton, nous montrons la persistance d'une courbe invariante indifférente pour une dynamique holomorphe fibrée de classe $C^{\infty}$. Une condition diophantienne sur la paire de nombres de rotation est demandée. On montre également que cette condition est optimale.

Abstract (On the persistence of invariant curves for smooth fibred holomorphic dynamics)

We establish the persistence of an invariant curve for a smooth fibered holomorphic dynamical system, provided that a diophantine condition for the pair of rotation numbers holds. We also show that this diophantine condition is optimal. Our proof relies on Hamilton's implicit function Theorem.
\end{abstract}

\section{Introduction}

La théorie des systèmes dynamiques unidimensionnels est loin d'être un champ épuisé. Cependant, au moins deux types de telles dynamiques peuvent être considérés comme étant bien compris. Il s'agit de la théorie des homéomorphismes positifs du cercle (une dimension réelle, voir par exemple [8], [32])

Texte reçu le 9 octobre 2007, révisé le 12 mai 2008 et le 11 juillet 2008, accepté le 12 août 2008

Mario Ponce, Pontificia Universidad Católica de Chile

Classification mathématique par sujets (2000). - 37F50, 37J40.

Mots clefs. - Dynamique holomorphe fibrée, condition diophantienne, courbes invariantes. 
et la dynamique semi-locale des germes holomorphes irrationnellement indifférents qui fixent l'origine du plan complexe (une dimension complexe, voir par exemple [4], [31]). On dit que de telles dynamiques, dont le comportement des orbites est contrôlé par un nombre réel qu'on appelle le nombre de rotation, sont de type elliptique (récurrentes, conservatives). On sait que lorsque ce nombre de rotation vérifie une certaine hypothèse arithmétique alors la dynamique est plutôt simple (peu de mesures invariantes, conjuguée à une dynamique linéaire, etc.). Par contre, lorsqu'il s'agit de dynamiques d'allure elliptique en dimension supérieure la théorie est beaucoup moins développée. Dans ce travail, nous présentons des résultats pour une classe de systèmes dynamiques qui ont une allure elliptique, qui ne sont pas unidimensionnels et qu'on peut considérer comme étant de dimension intermédiaire. Ces objets sont une généralisation non triviale des germes holomorphes. Définissons alors nos objets d'étude : soit $\alpha$ un nombre irrationnel et soit $U \subset \mathbb{C}$ un ouvert simplement connexe du plan complexe. Nous considérons des transformations fibrées de la forme

$$
\begin{aligned}
F: \mathbb{T}^{1} \times U & \longrightarrow \mathbb{T}^{1} \times \mathbb{C} \\
(\theta, z) & \longmapsto(\theta+\alpha, f(\theta, z)),
\end{aligned}
$$

où $\mathbb{T}^{1}$ est le cercle $\mathbb{R} / \mathbb{Z}$ et pour tout $\theta \in \mathbb{T}^{1}$ la fonction $f(\theta, \cdot): U \rightarrow \mathbb{C}$ est une fonction univalente (holomorphe et injective). Nous appelons une telle transformation $F$ une dynamique holomorphe fibrée au dessus du cercle et la noterons désormais $d h f$. Dans ce travail nous allons traiter avec des $d h f$ qui sont de classe $C^{\infty}$, dites dhf lisses.

Ce type de transformations s'inscrit dans le cadre plus général des produits croisés (skew-products). Les produits croisés engendrés par des dynamiques qui sont bien comprises ont attiré un grand intérêt ces dernières années car d'une part ils présentent des phénomènes nouveaux (voir [10], [30], etc.), et d'autre part, ils représentent un état intermédiaire entre les dynamiques unidimensionnelles et les dynamiques de dimension supérieure. Dans le cas des deux types de dynamiques elliptiques unidimensionnelles citées ci-dessus, la théorie la mieux développée est celle des homéomorphismes du cercle fibré au dessus d'une rotation irrationnelle. L'article de M. Herman [10] pose les bases de l'étude des homéomorphismes fibrés en définissant le nombre de rotation fibré. Plus récemment, cette étude a été relancée notamment par les travaux de G.Keller, T. Jäger et J. Stark (voir [29], [13]), qui ont établi en particulier une classification à la Poincaré de telles dynamiques basée sur les propriétés des nombres de rotation associés et l'existence de courbes invariantes. Dans sa thèse de doctorat, O. Sester [27] (voir aussi [28]) a étudié la dynamique des polynômes fibrés, en généralisant les notions classiques d'ensemble de Julia, fonction de Green et de la cardioïde principale de l'ensemble de Mandelbrot dans l'espace de paramètres. Les travaux de M. Jonsson (voir [15], [16]) sont

TOME $138-2010-\mathrm{N}^{\circ} 2$ 
aussi une référence importante sur la dynamique fibrée des transformations rationnelles. La réductibilité des cocycles quasipériodiques est un important sujet d'étude qui doit être considéré au moment de regarder les dynamiques fibrées au dessus d'une rotation irrationnelle (voir par exemple [19], [1]). En fait, R.Johnson and J.Moser [14] ont défini un nombre de rotation fibré pour l'opérateur de Schrödinger quasipériodique.

Il y a encore une autre raison pour laquelle les $d h f$ présentent un intérêt d'étude. Le problème qui nous traitons dans ce travail présente des caractéristiques très similaires à celles du problème bien connu de Melnikov (voir [22], [6], [3]). La persistance des tores invariants de dimension non-maximale pour les dynamiques hamiltoniennes présente au niveau infinitésimal (et a posteriori au niveau arithmétique) les mêmes ingrédients que la persistance des courbes invariantes pour les $d h f$.

La notion de point fixe ou de point périodique pour une transformation fibrée au dessus d'une rotation minimale n'a aucun sens (car $\alpha$ est un nombre irrationnel). L'extension naturelle de ce concept dans notre cadre est celle d'une courbe $u: \mathbb{T}^{1} \rightarrow \mathbb{C}$ invariante, c'est-à-dire une courbe de classe $C^{\infty}$ qui satisfait l'équation

$$
F(\theta, u(\theta))=(\theta+\alpha, u(\theta+\alpha))
$$

pour tout $\theta \in \mathbb{T}^{1}$, ou de façon équivalente $f(\theta, u(\theta))=u(\theta+\alpha)$. Ces objets jouent le rôle d'un centre autour duquel la dynamique de $F$ s'organise, en généralisant le rôle d'un point fixe pour la dynamique locale d'un germe holomorphe $g:(\mathbb{C}, 0) \rightarrow(\mathbb{C}, 0)$. En fait, dans [23] l'auteur étudie la dynamique locale autour d'une courbe invariante et montre un théorème de Siegel dans ce contexte.

Nous nous intéressons à l'existence d'une telle courbe invariante. Comme le nombre de rotation transversal (cf. définition 5) contrôle la dynamique locale, nous nous concentrons sur l'existence des courbes invariantes indifférentes avec un nombre de rotation transversal fixé. Dans ce travail nous traitons le problème de la persistance des courbes, sous des petites perturbations sur la dynamique. Nous obtenons un résultat du type KAM qui montre que la persistance a lieu sous certaines hypothèses arithmétiques sur la paire des nombres de rotation associée. Ainsi, le problème est un problème de petits diviseurs. Dans la classe analytique, nous pouvons obtenir des résultats beaucoup plus intéressants. En fait, dans [24] l'auteur montre que la persistance a lieu sous une hypothèse plus faible à la Brjuno sur la paire de nombres de rotation.

Remerciements. - Ce travail fait partie de la Thèse de Doctorat de l'auteur, préparée au sein du Laboratoire de Mathématiques d'Orsay. L'auteur voudrait remercier Jean-Christophe Yoccoz par sa direction et soutien constant, et remercie aussi Raphaël Krikorian pour ses corrections et suggestions. Cet article a été préparé pendant un séjour post-doctoral à l'Universidad Católica de Chile, 
supporté par le Proyecto ADI 17 Anillo en Sistemas Dinámicos en baja dimensión et le Proyecto FONDECYT Post-doctorado 3080055.

\section{Conditions arithmétiques et l'équation linéarisée}

Dans cette section nous allons d'abord rappeler quelques définitions classiques sur les conditions arithmétiques. Nous allons introduire des conditions arithmétiques adaptées aux problèmes de ce travail et nous traiterons aussi des équations linéaires dites cohomologiques qui font appel à ces conditions.

2.1. Conditions arithmétiques. - Soit $x$ un nombre réel. On pose $\|x\|=$ $\min _{p \in \mathbb{Z}}|x-p|$ la distance au plus proche entier (ou bien la distance à l'origine 0 dans $\mathbb{T}^{1}$ mesurée sur le cercle).

2.1.1. Conditions arithmétiques sur un nombre réel. - Un traitement plus complet peut se trouver dans plusieurs textes, dont [20], [5], [25]. Soient $c>0$, $\tau \geq 0$. Nous définissons les ensembles de nombres réels suivants

$$
\begin{aligned}
& \mathbb{C D}(c, \tau)=\left\{\alpha \in \mathbb{T}^{1} \backslash \mathbb{Q} \mid \forall N \geq 1 \quad\|N \alpha\| \geq \frac{c}{N^{1+\tau}}\right\} \\
& \mathbb{C D}(\tau)=\bigcup_{c>0} \mathbb{C D}(c, \tau) \\
& \mathbb{C D} \quad=\bigcup_{\tau \geq 0} \mathbb{C D}(\tau) .
\end{aligned}
$$

Si $\alpha$ appartient à $\mathbb{C D}$ on dit que $\alpha$ vérifie une condition diophantienne. Pour tout $\tau>0$ l'ensemble $\mathbb{C D}(\tau)$ est de mesure pleine au sens de Lebesgue. D'autre part, les ensembles $\mathbb{C D}(\tau)$ peuvent s'écrire comme une union dénombrable d'ensembles fermés et d'intérieur vide, ce qui implique que ces ensembles sont petits du point de vue de la topologie (catégorie de Baire).

2.1.2. Conditions arithmétiques sur une paire. — Soient $\alpha, \beta$ des nombres réels. Soient $c>0, \tau \geq 0$. Nous définissons les ensembles suivants

$$
\begin{aligned}
& \mathbb{C D}_{1}(c, \tau)=\left\{(\alpha, \beta) \in \mathbb{T}^{1} \times \mathbb{T}^{1} \mid \alpha \in \mathbb{C D} \text { et } \forall N \in \mathbb{Z} \quad\|N \alpha-\beta\| \geq \frac{c}{N^{1+\tau}}\right\} \\
& \mathbb{C D}_{1}(\tau)=\bigcup_{c>0} \mathbb{C D}_{1}(c, \tau) \\
& \mathbb{C D}_{1}=\bigcup_{\tau \geq 0} \mathbb{C D}_{1}(\tau) .
\end{aligned}
$$

Soit $\alpha \in \mathbb{C D}$. L'ensemble $\mathbb{C D}_{1}^{\alpha}(c, \tau)$ est l'ensemble de tous les $\beta$ tels que la paire $(\alpha, \beta)$ appartient à $\mathbb{C D}_{1}(c, \tau)$. De façon analogue on définit $\mathbb{C D}_{1}^{\alpha}(\tau), \mathbb{C D}_{1}^{\alpha}$. Pour tout $\tau>0$ l'ensemble $\mathbb{C D}_{1}^{\alpha}(\tau)$ est de mesure pleine. De plus, l'ensemble $\mathbb{C D}_{1}^{\alpha}(c, \tau)$ est un fermé d'intérieur vide. Les ensembles $\mathbb{C D}_{1}^{\alpha}(\tau), \mathbb{C D}_{1}^{\alpha}$ s'écrivent comme réunions dénombrables d'ensembles du type $\mathbb{C D}_{1}^{\alpha}(c, \tau)$, et donc, ils sont maigres, en particulier leur complémentaire est dense dans le cercle. 
2.2. Équation linéarisée. - Dans cette section nous allons rappeler des faits bien connus sur l'équation cohomologique, nous renvoyons le lecteur aux textes [8], [17]. Soit $\alpha$ dans $\mathbb{R} \backslash \mathbb{Q}$. Soit $\phi: \mathbb{T}^{1} \rightarrow \mathbb{C}$ une fonction de classe $C^{\infty}$ de moyenne $\int_{\mathbb{T}^{1}} \phi(\theta) d \theta$ nulle pour la mesure de Lebesgue $d \theta$ du cercle. Nous cherchons une solution $\psi: \mathbb{T}^{1} \rightarrow \mathbb{C}$ de classe $C^{\infty}$ à l'équation cohomologique classique

$$
\psi(\theta+\alpha)-\psi(\theta)=\phi(\theta) .
$$

Notons que la condition sur la moyenne de $\phi$ est nécessaire. Une éventuelle solution ne sera pas unique, puisque en ajoutant une constante nous obtenons d'autres solutions. Cependant, deux solutions à cette équation différent seulement d'une constante. La série de Fourier de $\phi$

$$
\mathcal{F}(\phi)(\theta)=\sum_{n \in \mathbb{Z} \backslash\{0\}} \hat{\phi}(n) e^{2 \pi i n \alpha}, \quad \hat{\phi}(n)=\int_{\mathbb{T}^{1}} \phi(\theta) e^{-2 \pi i n \theta} d \theta
$$

coïncide avec $\phi$ (on a convergence uniforme de toutes les dérivées). Soit $\psi$ une solution de classe $C^{\infty}$ à l'équation (1). On a donc

$$
\begin{aligned}
\mathcal{F}\left(\psi \circ R_{\alpha}-\psi\right) & =\mathcal{F}(\phi) \\
\hat{\psi}(n)\left(e^{2 \pi i n \alpha}-1\right) & =\hat{\phi}(n)
\end{aligned}
$$

pour tout $n \in \mathbb{Z} \backslash\{0\}$. Ceci nous suggère (et oblige) à définir une solution par la formule

$$
\psi(\theta)=\sum_{n \in \mathbb{Z} \backslash\{0\}} \frac{\hat{\phi}(n)}{e^{2 \pi i n \alpha}-1} e^{2 \pi i n \theta} .
$$

Notons que la valeur de la moyenne $\int_{\mathbb{T}^{1}} \psi(\theta) d \theta$ n'est pas fixée à priori et nous choisissons la normalisation $\hat{\psi}(0)=0$. Même si $\phi$ est de classe $C^{\infty}$, et donc ses coefficients de Fourier décroissent convenablement, la présence du facteur $\left(e^{2 \pi i n \alpha}-1\right)^{-1}$, qui peut devenir très grand si $n \alpha$ s'approche d'un entier, peut faire que cette série ne corresponde pas à celle d'une fonction de classe $C^{\infty}$. Plus précisément on a l'inégalité

$$
4\|n \alpha\| \leq\left|e^{2 \pi i n \alpha}-1\right| \leq 2 \pi\|n \alpha\| .
$$

On voit ainsi que les conditions arithmétiques de $\alpha$ apparaissent dans la discussion.

Proposition 2.1. - Si $\alpha \in \mathbb{C D}$ et $\phi$ est de classe $C^{\infty}$ alors la série (2) définit une solution de classe $C^{\infty}$ à l'équation (1). Si $\alpha \notin \mathbb{C D}$ alors il existe $\phi$ de classe $C^{\infty}$ telle que la série (2) n'est même pas une distribution. 
Soit $\beta \in \mathbb{R}$ et soit $\phi: \mathbb{T}^{1} \rightarrow \mathbb{C}$ une fonction de classe $C^{\infty}$. Dans ce travail nous devront considérer l'équation cohomologique tordue elliptiquement

$$
\tilde{\psi}(\theta+\alpha)-e^{2 \pi i \beta} \tilde{\psi}(\theta)=\tilde{\phi}(\theta) .
$$

En appliquant la méthode des séries de Fourier nous obtenons la série

$$
\tilde{\psi}(\theta)=\sum_{n \in \mathbb{Z}} \frac{\hat{\tilde{\phi}}(n)}{e^{2 \pi i n \alpha}-e^{2 \pi i \beta}} e^{2 \pi i n \theta} .
$$

Nous voyons que cette fois les petits diviseurs qui apparaissent sont de la forme $\|n \alpha-\beta\|$, et ainsi les conditions arithmétiques sur la paire $(\alpha, \beta)$ entrent dans la discussion. Notons cependant que si la paire $(\alpha, \beta)$ est rationnellement indépendante, la série (4) définit tous les coefficients de Fourier de la solution (ce qui ne se passait pas dans le cas de l'équation cohomologique classique pour le coefficient d'ordre 0).

Proposition 2.2. - Si $(\alpha, \beta) \in \mathbb{C D}_{1}$ et $\tilde{\phi}$ est de classe $C^{\infty}$ alors la série (4) définit une solution de classe $C^{\infty}$ à l'équation (3). Si $\beta \notin \mathbb{C D}_{1}^{\alpha}$ alors il existe $\tilde{\phi}$ de classe $C^{\infty}$ telle que la série (4) n'est même pas une distribution. De plus, étant donnés $\varepsilon>0, r \in \mathbb{N}$ on peut choisir $\tilde{\phi}$ de façon que sa taille $C^{r}$ soit plus petite que $\varepsilon$.

REMARQUE 2.3. - Cette proposition reste encore valable même si $\alpha$ n'appartient pas à $\mathbb{C D}$.

\section{Définitions et présentation du problème}

Dans ce travail nous allons supposer que les $d h f$ ainsi que les courbes invariantes sont toujours de classe $C^{\infty}$. Une courbe invariante $u: \mathbb{T}^{1} \rightarrow \mathbb{C}$ est indifférente si

$$
\int_{\mathbb{T}^{1}} \log \left|\partial_{z} f(\theta, u(\theta))\right| d \theta=0
$$

Notons que les fonctions $f(\theta, \cdot)$ sont injectives et $\partial_{z} f(\theta, \cdot)$ ne s'annule donc pas. Nous traiterons la persistance des courbes indifférentes de degré nul, c'està-dire, les courbes invariantes indifférentes pour lesquelles on a l'hypothèse suivante : le degré topologique de l'application

$$
\theta \longmapsto \partial_{z} f(\theta, u(\theta))
$$

est nul. De façon équivalente, l'application ci-dessus est homotope à une constante dans $\mathbb{C} \backslash\{0\}$. Notons que dans ce cas l'application $\log \partial_{z} f(\theta, u(\theta))$ est bien définie, $\bmod 2 \pi$. 
3.1. Le nombre de rotation transversal. - À une courbe invariante indifférente de degré nul nous associons un nombre qui mesure la vitesse moyenne d'enroulement des orbites proches autour de la courbe invariante :

$$
\varrho_{t r}(u)=\frac{1}{2 \pi i} \int_{\mathbb{T}^{1}} \log \partial_{z} f(\theta, u(\theta)) d \theta .
$$

Cette quantité est bien définie mod 1 et nous l'appelons le nombre de rotation transversal. Par exemple, la dhf donné par $F(\theta, z)=\left(\theta+\alpha, e^{2 \pi i \beta} z\right)$ avec $\beta$ un nombre réel dans l'interval $[0,1)$, est la plus simple mais pas la moins intéressante. La courbe $u=\{z \equiv 0\}_{\theta \in \mathbb{T}^{1}}$ est lisse, invariante, de degré nul, indifférente et $\varrho_{t r}(u)=\beta$.

3.2. Forme normale. - Soit $F$ une dynamique holomorphe fibrée avec une courbe invariante $\left\{u_{0}(\theta)\right\}_{\theta \in \mathbb{T}^{1}}$ indifférente de classe $C^{\infty}$, de degré nul et $\varrho_{t r}\left(u_{0}\right)=\beta \in \mathbb{R}$. Supposons que nous pouvons résoudre l'équation cohomologique

$$
\frac{u_{1}(\theta+\alpha)}{u_{1}(\theta)} e^{2 \pi i \beta}=\partial_{z} f\left(\theta, u_{0}(\theta)\right)
$$

avec $u_{1}$ une fonction à valeurs dans $\mathbb{C}$, qui ne s'annule pas, de degré nul et de classe $C^{\infty}$. En faisant le changement de coordonnées

$$
H(\theta, z)=\left(\theta, u_{0}(\theta)+u_{1}(\theta) z\right)
$$

nous obtiendrons une forme normale pour $F, \tilde{F}=H \circ F \circ H^{-1}$,

$$
(\theta, z) \stackrel{\tilde{F}}{\longmapsto}\left(\theta+\alpha, e^{2 \pi i \beta} z+\rho(\theta, z)\right),
$$

où la fonction $\rho(\theta, z)$ est définie dans le produit du cercle par un voisinage de l'origine complexe, est de classe $C^{\infty}$ et s'annule jusqu'à l'ordre 2 en $z=0$. On verra que l'équation (6) peut être résolue sous une hypothèse arithmétique diophantienne sur le nombre $\alpha$ (cf. section 2).

La discussion précédente nous permet donc de dire que la dynamique holomorphe fibrée autour d'une courbe invariante peut être vue, à la résolution d'une équation cohomologique près, comme une dynamique fibrée par des germes holomorphes qui fixent l'origine, avec un nombre de rotation bien précis, au dessus d'une rotation irrationnelle du cercle. Dans ce travail cela sera le cas, on supposera $\alpha \in \mathbb{C D}$.

3.3. Petites perturbations. - Ce travail est consacré à l'étude de la persistance d'une courbe invariante $u$ (lisse, de degré nul et indifférente), sous des petites 
perturbations sur la dynamique. D'après la discussion précédente, sous l'hypothèse diophantienne sur $\alpha$, nous disons que $\tilde{F}$ est une petite perturbation de $F$ autour de la courbe si, dans les coordonnées de $H$, la transformation $\tilde{F}$ s'écrit

$$
(\theta, z) \stackrel{\tilde{F}}{\longmapsto}\left(\theta+\alpha, \tilde{\rho}_{0}(\theta)+\left(\tilde{\rho_{1}}(\theta)+e^{2 \pi i \varrho_{t r}(u)}\right) z+\tilde{\rho}(\theta, z)\right)
$$

avec $\tilde{\rho}_{0}, \tilde{\rho}_{1}: \mathbb{T}^{1} \rightarrow \mathbb{C}$ des fonctions de classe $C^{\infty}$ de taille petite (dans une topologie adéquate), et avec $\tilde{\rho}(\theta, \cdot)$ holomorphe dans un disque $D_{r}, r>0$, s'annulant à l'ordre 2 en $z=0$. La taille de $\tilde{\rho}$ est comparable à la taille de $\rho$ (dans un sens à préciser). Ces hypothèses de proximité entre la perturbation et la transformation originale seront bien précisées dans l'énoncé du théorème principal. Notons que nous considérons des perturbations qui changent seulement la partie holomorphe des dynamiques. Le nombre de rotation sur la base $\alpha$ est donc fixé.

3.4. Familles à un paramètre. - Dans les résultats de type KAM, une perturbation sur une dynamique elliptique donne lieu aussi à une perturbation sur les fréquences concernées, qui sont les valeurs contrôlant la dynamique. Donc, nous ne pouvons pas espérer obtenir les mêmes phénomènes dynamiques que dans la situation non perturbée. Pour obtenir une persistance de ces phénomènes on introduit une famille de perturbations à 1 paramètre qui cherche à corriger les fréquences par rapport à la valeur originale, et à pouvoir ainsi se référer à un modèle avec des bonnes fréquences (en général les fréquences de référence vérifient une hypothèse arithmétique). De cette façon on montre que, quitte à corriger les fréquences, plusieurs propriétés dynamiques sont persistantes (linéarisation, existence d'objets invariants, etc.). On dit que la persistance est en co-dimension 1 (voir [2]).

Dans notre travail nous perturbons seulement la partie holomorphe de la transformation. Donc la fréquence correspond au nombre de rotation transversal, qui est une donnée de 1 dimension complexe. Soit $F$ une fhd et $u$ une courbe invariante indifférente, avec $\varrho_{t r}(u)=\beta \in \mathbb{R}$. Soit $\Sigma$ un ouvert de $\mathbb{C}$. Une petite perturbation transverse de $F$ est une famille à 1 paramètre complexe $\left\{F_{t}\right\}_{t \in \Sigma}$ de $f h d$ (une courbe complexe dans l'espace des fhd) qui vérifie les hypothèses suivantes : chaque élément $F_{t}$ est une petite perturbation de $F$ et le nombre de rotation transversal (même quand la courbe n'existe pas) bouge avec la famille $\left\{F_{t}\right\}_{t \in \Sigma}$ (voir le théorème 4.1 pour une définition précise). Nous disons que la courbe $u$ est persistante si pour toute petite perturbation transverse $\left\{F_{t}\right\}_{t \in \Sigma}$ il existe un paramètre $t^{*} \in \Sigma$ tel que $F_{t^{*}}$ possède une courbe invariante indifférente $u^{*}$ avec $\varrho_{t r}\left(u^{*}\right)=\beta$. En gros, le principal résultat de ce travail dit que, modulo une petite correction complexe, les courbes invariantes indifférentes sont persistantes en classe $C^{\infty}$ pourvu que les nombres de rotation vérifient une condition arithmétique de type diophantienne.

TOME $138-2010-\mathrm{N}^{\mathrm{O}} 2$ 


\section{4. Énoncé du théorème}

Nous considérons une famille de $\operatorname{dhf}\left\{F_{s}\right\}_{s \in \Sigma \subset \mathbb{C}}$ à un paramètre complexe $s \in \Sigma \subset \mathbb{C}$, où chaque $F_{s}$ est une $d h f$ de classe $C^{\infty}$. Pour $\beta \in \mathbb{R}$ nous fixons la notation $\lambda=e^{2 \pi i \beta}$. Nous disons qu'une telle famille est lisse si, écrite sous la forme habituelle

$$
\begin{aligned}
F_{s}(\theta, z) & =\left(\theta+\alpha, f_{s}(\theta, z)\right) \\
& =\left(\theta+\alpha, \rho_{0, s}(\theta)+\rho_{1, s}(\theta) z+\lambda z+\rho_{s}(\theta, z)\right),
\end{aligned}
$$

les fonctions $(s, \theta, z) \mapsto\left(\rho_{0, s}(\theta), \rho_{1, s}(\theta), \rho_{s}(\theta, z)\right)$ sont des fonctions de classe $C^{\infty}$. Les $\rho_{s}(\theta, \cdot)$ sont holomorphes, continues jusqu'au bord $\partial \mathbb{D}$ et s'annulent jusqu'au l'ordre 2 en $z=0$ pour pour tout $\theta$ dans $\mathbb{T}^{1}$ et tout $s$ dans $\Sigma$. Dans ce travail nous allons désigner une famille de $d h f$ soit par $\left\{F_{s}\right\}$, soit par $\left\{f_{s}\right\}$ où $f_{s}$ représente la partie holomorphe de la dynamique $F_{s}$. Nous disons que $F_{s}$ est la $d h f$ associée à $f_{s}$. Nous appellerons également lisse $\left(C^{\infty}\right)$ une famille de parties holomorphes $\left\{f_{s}\right\}_{s \in \Sigma \subset \mathbb{C}}$. Avant d'énoncer le résultat principal de ce travail nous allons introduire quelques notations.

4.1. Des notations et normes considérées. - Nous allons écrire $f^{\prime}(\theta, z)$ au lieu de $\partial_{z} f(\theta, z)$ et $f^{(i)}(\theta, z)$ pour les dérivées d'ordre supérieur $\partial_{z}^{i} f(\theta, z)$. Pour une fonction $g: \mathbb{T}^{1} \rightarrow\left(B,|\cdot|_{B}\right)$ de classe $C^{\infty}$, où $B$ est un espace de Banach avec norme $|\cdot|_{B}$, nous considérons la norme $C^{0}$ et $C^{r}$ pour $r \in \mathbb{N}$ comme étant

$$
\|g\|_{0}=\sup _{\theta \in \mathbb{T}^{1}}|g(\theta)|_{B}, \quad\|g\|_{r}=\sum_{i=0}^{r}\left\|\frac{\partial^{i} g}{\partial \theta^{i}}\right\|_{0} .
$$

L'espace de Banach que nous aurons toujours en tête sera l'espace $\Omega(\overline{\mathbb{D}})$ des fonctions holomorphes du disque unité complexe, qui sont continues jusqu'au bord, muni de sa norme sup. Pour une matrice $A: \mathbb{R}^{2} \rightarrow \mathbb{R}^{2}$ nous allons noter

$$
\|A\|_{\mathfrak{L}}=\sup _{|v|=1}|A v|, \quad[A]_{\mathfrak{L}}=\inf _{|v|=1}|A v|
$$

sa norme usuelle et la plus petite valeur propre respectivement, où $|\cdot|$ est une norme dans $\mathbb{R}^{2}$.

Lemma 4.1. - Soit $A: \mathbb{R}^{2} \rightarrow \mathbb{R}^{2}$ une matrice avec $[A]_{\mathfrak{L}} \neq 0$. Alors la matrice $A$ est inversible et la norme de la matrice inverse $A^{-1}$ vérifie

$$
\left\|A^{-1}\right\|_{\mathfrak{L}} \leq[A]_{\mathfrak{L}}^{-1} .
$$

ThÉORÈme 4.1 . - Pour toute paire $(\alpha, \beta)$ qui vérifie l'hypothèse $\mathbb{C D}_{1}$ et pour toutes constantes $L>1, M>1$ il existe $\tilde{\varepsilon}>0$ qui dépend de $L, M,(\alpha, \beta)$, un nombre entier naturel $r \geq 2$ qui dépend de la paire $(\alpha, \beta)$ tels que, si une famille à un paramètre complexe $\left\{f_{s}\right\}_{s \in \Sigma}$ de fonctions de $\mathbb{T}^{1}$ vers $\Omega(\overline{\mathbb{D}})$ vérifie pour un certain $\varepsilon$ dans $(0, \tilde{\varepsilon}]$ 
1. $\left\|\rho_{0, s}\right\|_{r} \leq 10 \varepsilon$ pour tout $s$ dans $D(0,2 L \varepsilon) \subset \Sigma$

2. $\left\|\rho_{1, s}\right\|_{r} \leq 10 \varepsilon$ pour tout $s$ dans $D(0,2 L \varepsilon) \subset \Sigma$

3. $\left[\left.\partial_{s} \int_{\mathbb{T}^{1}} \rho_{1, t}(\theta) d \theta\right|_{s=0}\right]_{\mathfrak{L}}>L^{-1} ;\left\|\rho_{1, s=0}\right\|_{r} \leq \varepsilon$

4. $\left\|\partial_{s}^{2} \rho_{1, s}\right\|_{0}+\left\|\partial_{s} \rho_{s}\right\|_{0}+\left\|\rho_{s}\right\|_{r} \leq M$ pour tout $s$ dans $D(0,2 L \varepsilon) \subset \Sigma$

alors il existe un paramètre $s^{*}$ dans le disque $D(0,2 L \varepsilon) \subset \Sigma$ et une courbe $u: \mathbb{T}^{1} \rightarrow \mathbb{D}$ de classe $C^{\infty}$, indifférente, de degré nul, qui est invariante par la dynamique holomorphe fibrée $F_{s^{*}}(\theta, z)=\left(\theta+\alpha, f_{s^{*}}(\theta, z)\right)$, et son nombre de rotation transversal est $\varrho_{t r}(u)=\beta$.

Par exemple, prenons une fonction $f$ avec $\|f-\lambda z\|_{r} \leq \tilde{\varepsilon}$ et la famille à un paramètre $\left\{e^{s} f\right\}_{s \in \mathbb{C}}$. Pour la notation habituelle nous avons

$$
\begin{aligned}
\rho_{1, s} & =e^{s} f^{\prime}-\lambda \\
& =e^{s}\left(f^{\prime}-\lambda\right)+\lambda\left(e^{s}-1\right) \\
\partial_{s} \rho_{1, s} & =e^{s}\left(f^{\prime}-\lambda\right)+\lambda e^{s} .
\end{aligned}
$$

Avec $L=2$, nous avons bien toutes les hypothèses du théorème 4.1 vérifiées et donc aussi les conclusions. Cependant, le théorème ci dessus découlera d'une version plus faible qui est plus adaptée à la méthode de démonstration utilisée, et qui s'adresse justement a la famille de notre example,

ThÉORÈme 4.2. - Pour toute paire $(\alpha, \beta)$ qui vérifie l'hypothèse diophantienne $\mathbb{C D}_{1}$ il existe $\bar{\varepsilon}>0$ et un nombre naturel $r \geq 2$ tels que si une fonction $f: \mathbb{T}^{1} \rightarrow \Omega(\overline{\mathbb{D}})$ satisfait $\|f-\lambda z\|_{r}<\bar{\varepsilon}$ alors il existe $t$ dans $\mathbb{C}$ et une courbe $u: \mathbb{T}^{1} \rightarrow \mathbb{D}$ de classe $C^{\infty}$, indifférente, de degré nul, qui est invariante par la dynamique holomorphe fibrée $F^{*}(\theta, z)=\left(\theta+\alpha, e^{t} f\right)$, et son nombre de rotation transversal est $\varrho_{t r}(u)=\beta$.

Le théorème 4.2 est donc la version du théorème 4.1 pour le cas particulier de la famille $\left\{f_{t}\right\}_{t \in \mathbb{C}}=\left\{e^{t} f\right\}_{t \in \mathbb{C}}$ et $f$ proche de $\lambda z$. Les hypothèses 1., 2., 4. et 5 . traduisent le fait que $F_{s}$ est une petite perturbation d'une forme normale. L'hypothèse 3 . exprime le fait que la famille est transverse, au sens où le nombre de rotation transversal est calculé à l'aide de la valeur de $\rho_{1}$ et ce valeur varie avec le paramètre $s$.

Notons que la condition diophantienne demandée dans les hypothèses $\mathrm{du}$ théorème est exactement celle qui apparaît comme nécessaire et suffisante pour pouvoir toujours résoudre l'équation linéarisée associée au problème (voir section 2.2). Cette simple observation nous permet de montrer que la condition diophantienne est optimale pour le problème de la persistance de la courbe invariante dans le cas $C^{\infty}$ comme le montre la 
Proposition 4.2. - Soit $\alpha \in \mathbb{C D}$ et soit $\beta$ tel que la paire $(\alpha, \beta)$ ne satisfait pas la condition diophantienne $\mathbb{C D}_{1}$. Pour tous $\varepsilon>0, r$ dans $\mathbb{N}$ il existe une fonction $a: \mathbb{T}^{1} \rightarrow \mathbb{C}$ de classe $C^{\infty}$ et de norme $C^{r}$ plus petite que $\varepsilon$ telle que la famille à un paramètre complexe

$$
F_{t}(\theta, z)=(\theta+\alpha, t a(\theta)+t \lambda z)
$$

vérifie que pour tout $t$ dans $\mathbb{C}$ la dynamique holomorphe fibrée $F_{t}$ ne possède aucune courbe invariante de classe $C^{\infty}$ avec $\beta$ comme nombre de rotation transversal.

Démonstration. - Notons d'abord que pour une telle famille la dérivée par rapport à $z$ de la partie holomorphe est toujours égal à $t \lambda=t e^{2 \pi i \beta}$, donc le seul paramètre qui permet l'existence d'une courbe invariante avec nombre de rotation transversal égal à $\beta$ est $t=1$. L'hypothèse de transversalité est immédiate. Supposons que pour une fonction $a: \mathbb{T}^{1} \rightarrow \mathbb{C}$ de classe $C^{\infty}$ nous avons une courbe invariante $u: \mathbb{T}^{1} \rightarrow \mathbb{D}$ pour la dynamique holomorphe fibrée $F(\theta, z)=(\theta+\alpha, a(\theta)+\lambda z)$. Nous pouvons alors déterminer de façon unique cette courbe à l'aide de l'équation de la courbe invariante

$$
a(\theta)+e^{2 \pi i \beta} u(\theta)=u(\theta+\alpha)
$$

qui est bien une équation cohomologique comme celles étudiées dans la section 2.2. La proposition 2.2 nous permet construire une fonction $a$ de façon que l'unique courbe invariante solution à l'équation ci-dessus ne soit même pas une distribution.

\section{Le théorème des fonctions implicites de Hamilton}

Depuis les travaux de Sergeraert [26] et plus particulièrement ceux de Herman [11], [2], l'utilisation des théorèmes de fonctions implicites dans les espaces de Fréchet pour résoudre des problèmes dynamiques faisant intervenir des petits diviseurs s'est avérée très fructueuse. Cette technique repose sur le fait que la résolution du problème linéaire associé (en général une équation cohomologique) est fortement relié aux propriétés arithmétiques des fréquences impliquées (voir section 2.2). Rappelons qu'un théorème de fonctions implicites assure en général l'existence de solutions du problème non linéaire qui nous occupe pourvu que le problème linéaire admette des solutions.

Le théorème des fonctions implicites de Hamilton nous permet d'appliquer cette technique dans le cadre des fonctions de classe $C^{\infty}$, qui d'habitude nous amènent à travailler avec des espaces qui ne sont pas des espaces de Banach, mais des espaces de Fréchet. La différence essentielle avec les théorèmes des fonctions implicites classiques dans les espaces de Banach repose sur le fait que celui de Hamilton exige de résoudre le problème linéaire associé non pas 
seulement au point où la solution est connue, mais dans tout un voisinage de ce point. Les prochains paragraphes vont préciser tous les objets qui interviennent dans l'énoncé du théorème de Hamilton. Bien entendu, nous allons nous servir de ce théorème pour montrer la version faible du théorème de la persistance de la courbe invariante dans le cas $C^{\infty}$ (théorème 4.2). Nous renvoyons le lecteur aux articles [7], [2] pour un traitement plus détaillé du théorème de Hamilton.

5.1. Bons espaces de Fréchet. - Nous disons que l'espace vectoriel topologique $E$ est un bon espace de Fréchet au sens de Hamilton s'il existe une famille croissante de seminormes $\left\{\|\cdot\|_{i}\right\}_{i \in \mathbb{N}}$ qui définissent sa topologie, une famille d'opérateurs d'approximation et lissage $\left\{S_{t}\right\}_{t>1}$ et des constantes positives $C_{n, k}$ pour chaque paire $(n, k)$ dans $\mathbb{N}^{2}$ qui vérifient

1. $S_{t}: E \rightarrow E$ est une application linéaire continue.

2. Si $k \leq n, \forall x \in E, \forall t \in] 1,+\infty] \quad\left\{\begin{array}{l}\left\|S_{t}(x)\right\|_{n} \leq C_{n, k} t^{n-k}\|x\|_{k} \\ \left\|\left[I d-S_{t}\right](x)\right\|_{k} \leq C_{k, n} t^{k-n}\|x\|_{n} .\end{array}\right.$

Les dernières inégalités impliquent des inégalités de convexité sur les seminormes \|\|$_{i}$, (Hadamard) : pour chaque paire $(n, k)$ dans $\mathbb{N}^{2}$ il existe des constantes positives $\tilde{C}_{n, k}$ tels que

$$
\|x\|_{l} \leq \widetilde{C}_{k, n}\|x\|_{k}^{1-\alpha}\|x\|_{n}^{\alpha}
$$

pour tout $x$ qui appartient à $E$, pour tous les entiers $k \leq l \leq n$, où $\alpha$ est défini par $l=(1-\alpha) k+\alpha n$.

La somme directe (le produit) $E \oplus F$ de deux bons espaces de Fréchet est un bon espace de Fréchet avec les seminormes $\|(u, v)\|_{i_{E \oplus F}}=\|u\|_{i_{E}}+\|v\|_{i_{F}}$ et les opérateurs de lissage et approximation $S_{t}(u, v)=\left(S_{t}^{E} u, S_{t}^{F} v\right)$. Nous disons que l'application $f: U \subset E \rightarrow F$ d'un ouvert $U$ d'un bon espace de Fréchet $E$ vers un autre bon espace de Fréchet $F$, est une bonne application au sens de Hamilton si pour tout $x_{0}$ qui appartient à $U$ il existe un voisinage $V$ de $x_{0}$ dans $U$, un entier positif $r$ et pour tout $i \in \mathbb{N}$ des constantes positives $C_{i}$ tels que

$$
\|f(x)\|_{i} \leq C_{i}\left(1+\|x\|_{i+r}\right)
$$

pour tout $x$ dans $V$ et pour tout $i$ dans $\mathbb{N}$.

5.1.1. Différentiabilité au sens de Gâteaux. - Soient $E$ et $F$ deux espaces vectoriels topologiques, $U$ un ouvert de $E$ et $f$ une application de $U$ vers $F$. On dit que $f$ est de classe $C^{1}$ (au sens de Gâteaux) lorsque

1. $f$ est continue.

TOME $138-2010-\mathrm{N}^{\mathrm{O}} 2$ 
2. Il existe une application $D f: U \times E \rightarrow G$ continue, linéaire en la deuxième coordonnée et telle que pour tout $x$ dans $U, y$ dans $E$ on a

$$
\lim _{t \rightarrow 0} \frac{1}{t}\{f(x+t y)-f(x)\}=D f(x) y .
$$

Les applications de classe $C^{k}$ (au sens de Gâteaux) sont définies par récurrence sur $k$ : soit $k$ dans $\mathbb{N} \backslash\{0,1\}, f$ est dite de classe $C^{k}$ lorsqu'elle est de classe $C^{1}$ et que $D f$ est de classe $C^{k-1}$ sur l'ouvert $U \times E$ de $E \times E$. Nous disons que $f$ est une bonne application de classe $C^{k}(k$ dans $\mathbb{N} \cup\{\infty\})$ si $f$ est de classe $C^{k}$ au sens de Gâteaux, et que $f$ ainsi que ses dérivés jusque à l'ordre $k$ sont des bonnes applications (une telle dérivée $D^{i} f$ est une application à valeurs dans $F$ définie sur l'ouvert $U \times E^{i}$ de $\left.E^{i+1}\right)$.

Proposition 5.1. - Donnons nous trois bons espaces de Fréchet $E, F, G, U$ un ouvert de $E$ et $V$ un ouvert de F. Si $f: U \mapsto V$ et $g: V \mapsto G$ sont des bonnes applications de classe $C^{k}(k$ dans $\mathbb{N} \cup\{\infty\})$, alors la composition $g \circ f$ est une bonne application de classe $C^{k}$. La projection $E \times F \longrightarrow E$ est une bonne application de classe $C^{k}$ pour tout $k$.

\subsection{Théorème des fonctions implicites}

ThÉORÈme 5.1 (Fonction implicite). - Donnons nous trois bons espaces de Fréchet $E, F, G, U$ un ouvert de $E \times F, f: U \rightarrow G$ une bonne application de classe $C^{r}(2 \leq r \leq \infty)$ et $\left(x_{0}, y_{0}\right)$ qui appartient à $U$. Supposons qu'il existe un voisinage $V_{0}$ de $\left(x_{0}, y_{0}\right)$ et une bonne application continue et linéaire dans la deuxième coordonnée $L: V_{0} \times G \rightarrow F$ telle que si $(x, y)$ appartient à $V_{0}$ alors $D_{2} f(x, y)$ est inversible avec $L(x, y)$ comme son inverse. On en déduit alors que $x_{0}$ a un voisinage $W$ dans lequel est définie une bonne application de classe $C^{r}, g: W \rightarrow F$ telle que :

1. $g\left(x_{0}\right)=y_{0}$;

2. pour tout $x$ dans $W$ la paire $(x, g(x))$ appartient $\grave{a} U$ et on a $f(x, g(x))=$ $f\left(x_{0}, y_{0}\right)$

En plus, si $x$ appartient $\grave{a} W$, y est dans un petit voisinage au tour de $y_{0}$ et on a que $f(x, y)=f\left(x_{0}, y_{0}\right)$ si et seulement si $y=g(x)$.

\section{Preuve du théorème 4.2}

6.1. Les bons espaces de Fréchet $\Gamma^{\infty}\left(\mathbb{T}^{1}, B\right)$. - Nous définissons $\Gamma^{\infty}\left(\mathbb{T}^{1}, B\right)$ comme l'espace des fonctions $f: \mathbb{T}^{1} \rightarrow B$ de classe $C^{\infty}$ (au sens de Gâteaux) à valeurs dans un espace de Banach $\left(B,|\cdot|_{B}\right)$ et nous le munissons de la famille de seminormes $C^{r}$. Avec ces seminormes l'espace $\Gamma^{\infty}\left(\mathbb{T}^{1}, B\right)$ devient un espace de Fréchet. Nous définirons des opérateurs d'approximation et lissage qui en 
font un bon espace de Fréchet sur lequel nous pourrons appliquer le théorème 5.1 .

6.1.1. Opérateurs de lissage et approximation sur $\Gamma^{\infty}\left(\mathbb{T}^{1}, B\right)$ - — Les opérateurs que nous allons définir dans cette section sont classiques et nous renvoyons le lecteur aux articles [12], [9] pour les démonstrations. La seule chose à souligner dans notre cas est que l'espace d'arrivée des fonctions est un espace de Banach, où on peut définir, de la même façon que dans le cas réel ou complexe, l'intégrale de Riemann d'une fonction (voir [18]). L'opération * de convolution est ainsi bien définie. Par la suite nous identifierons les fonctions qui appartiennent à $\Gamma^{\infty}\left(\mathbb{T}^{1}, B\right)$ aux fonctions dans $C_{\mathbb{Z}}^{\infty}(\mathbb{R}, B)$, les fonctions de classe $C^{\infty}$ qui sont $\mathbb{Z}$-périodiques à valeurs dans $B$. Soit $\eta$ dans $C^{\infty}(\mathbb{R}, B)$ vérifiant $\operatorname{supp}(\eta) \subset[-1,1], \eta(-x)=\eta(x)$ et $\eta(x)=1$ si $|x| \leq \frac{1}{2}$. Soit $\phi(x)=\int_{\mathbb{R}} e^{-2 \pi i \xi x} \eta(\xi) d \xi ;$ on pose, pour $t \geq 1, \phi_{t}(x)=t \phi(t x)$. Pour $f$ dans $C_{\mathbb{Z}}^{\infty}(\mathbb{R}, B)$ on définit l'opérateur de lissage et approximation

$$
S_{t} f=f * \phi_{t}=\int_{\mathbb{R}} f(x-y) \phi_{t}(y) d y \quad \in \quad C_{\mathbb{Z}}^{\infty}(\mathbb{R}, B) .
$$

Par la formule d'inversion de Fourier, on a pour tout $v$ dans $B$ et pour tout $n$ dans $\mathbb{Z}$

$$
S_{t}\left(v e^{2 \pi i n \theta}\right)=v \eta\left(-\frac{n}{t}\right) e^{2 \pi i n \theta}
$$

donc $S_{t} f$ est un polynôme trigonométrique de degré au plus $|t|$. Les opérateurs $S_{t}$ ont les propriétés suivantes de lissage et approximation

Proposition 6.1. - Pour chaque paire $(k, n)$ dans $\mathbb{N}^{2}$ il existe des constantes positives $C_{k, n}$ telles que si $f$ appartient $\grave{a} \Gamma^{\infty}\left(\mathbb{T}^{1}, B\right)$ et $n \geq k$ alors pour tout $t \geq 1$

1. $\left\|S_{t} f\right\|_{n} \leq C_{k, n} t^{n-k}\|f\|_{k}$.

2. $\left\|S_{t} f-f\right\|_{k} \leq C_{n, k} t^{k-n}\|f\|_{n}$.

6.2. Quelques bonnes applications. - À partir des bons espaces de Fréchet $\Gamma^{\infty}\left(\mathbb{T}^{1}, B\right)$ définis dans la section 6.1 on définit ici les bons espaces de Fréchet que nous allons utiliser lors de la preuve du théorème 4.2 :

1. L'espace $C^{\infty}\left(\mathbb{T}^{1}, \mathbb{C}\right)$ des fonctions de classe $C^{\infty}$ de $\mathbb{T}^{1}$ vers l'espace de Banach $\mathbb{C}$ est le bon espace de Fréchet $\Gamma^{\infty}\left(\mathbb{T}^{1}, \mathbb{C}\right)$.

2. Le bon espace de Fréchet $\Gamma^{\infty}\left(\mathbb{T}^{1}, \Omega(\overline{\mathbb{D}})\right)$ est l'espace des fonctions de classe $C^{\infty}$ de $\mathbb{T}^{1}$ vers l'espace de Banach $\Omega(\overline{\mathbb{D}})$. Ceci est l'espace des parties holomorphes des dynamiques holomorphes fibrées de classe $C^{\infty}$. Rappelons que l'espace de Banach $\Omega(\overline{\mathbb{D}})$ est l'espace des fonction holomorphes sur $\mathbb{D}$ qui sont continues jusqu'au bord, avec la norme $|f|_{\Omega(\overline{\mathbb{D}})}=\sup _{|z|<1}|f(z)|$. 
Lemma 6.2. - Soit l'ensemble ouvert $A=\left\{u \in C^{\infty}\left(\mathbb{T}^{1}, \mathbb{C}\right) \mid\|u\|_{0}<1 / 2\right\}$ de $C^{\infty}\left(\mathbb{T}^{1}, \mathbb{C}\right)$. Les applications

1.

$$
\begin{aligned}
\Gamma^{\infty}\left(\mathbb{T}^{1}, \Omega(\overline{\mathbb{D}})\right) & \longrightarrow \Gamma^{\infty}\left(\mathbb{T}^{1}, \Omega(\overline{D(0,3 / 4)})\right) \\
f & \longmapsto f^{(i)}=\frac{\partial^{i} f}{\partial z^{i}}
\end{aligned}
$$

2.

$$
\begin{aligned}
C^{\infty}\left(\mathbb{T}^{1}, \mathbb{C}\right) \times C^{\infty}\left(\mathbb{T}^{1}, \mathbb{C}\right) & \longrightarrow C^{\infty}\left(\mathbb{T}^{1}, \mathbb{C}\right) \\
(u, v) & \longmapsto u v \\
(u, v) & \longmapsto u+v,
\end{aligned}
$$

3.

$$
\begin{aligned}
C^{\infty}\left(\mathbb{T}^{1}, \mathbb{C}\right)_{0} & \longrightarrow C^{\infty}\left(\mathbb{T}^{1}, \mathbb{C}\right)_{0} \\
v & \longmapsto v^{-1} \\
v & \longmapsto e^{v}
\end{aligned}
$$

où $C^{\infty}\left(\mathbb{T}^{1}, \mathbb{C}\right)_{0}$ est l'espace ouvert de $C^{\infty}\left(\mathbb{T}^{1}, \mathbb{C}\right)$ des fonctions qui ne s'annulent pas,

4 .

$$
\begin{aligned}
C^{\infty}\left(\mathbb{T}^{1}, \mathbb{C}\right)_{d_{0}} & \longrightarrow C^{\infty}\left(\mathbb{T}^{1}, \mathbb{C}\right) \\
h & \longmapsto \log h
\end{aligned}
$$

où $C^{\infty}\left(\mathbb{T}^{1}, \mathbb{C}\right)_{d_{0}}$ est l'espace ouvert de $C^{\infty}\left(\mathbb{T}^{1}, \mathbb{C}\right)$ des fonctions de degré nul,

5 .

$$
\begin{aligned}
C^{\infty}\left(\mathbb{T}^{1}, \mathbb{C}\right) & \longrightarrow \mathbb{C} \\
v & \longmapsto \int_{\mathbb{T}^{1}} v(\theta) d \theta,
\end{aligned}
$$

6. l'application $\eta_{r}$

$$
\begin{aligned}
\Gamma^{\infty}\left(\mathbb{T}^{1}, \Omega(\overline{D(0, r)})\right) \times A & \stackrel{\eta_{r}}{\longrightarrow} C^{\infty}\left(\mathbb{T}^{1}, \mathbb{C}\right) \\
(f, u) & \longmapsto f(\cdot, u(\cdot))
\end{aligned}
$$

pour tout $r$ dans $(1 / 2,1]$,

sont de bonnes applications au sens de Hamilton.

Démonstration. - Le point 1. est une conséquence des estimations de Cauchy. Les points 2., 3., 4., 5., sont classiques et en fait ce sont des bonnes applications de classe $C^{\infty}$. Nous montrerons par la suite le point 6 . Rappelons que le fait d'être une bonne application est un fait local, donc étant donnée 
$(\bar{f}, \bar{u})$ dans $\Gamma^{\infty}\left(\mathbb{T}^{1}, \Omega(\overline{\mathbb{D}})\right) \times A$ nous fixons un voisinage borné $C^{0}$ de $\bar{f}$ dans $\Gamma^{\infty}\left(\mathbb{T}^{1}, \Omega(\overline{D(0, r)})\right)$ et un voisinage $C^{0}$ de $\bar{u}$ dans $A \subset C^{\infty}\left(\mathbb{T}^{1}, \mathbb{C}\right)$. Notons que les estimations de Cauchy assurent l'existence pour chaque paire $(i, j)$ dans $\mathbb{N}^{2}$, de constantes positives $C_{i, j}$ telles que

$$
\left\|\frac{\partial^{j}}{\partial \theta^{j}} f^{(i)}(\theta, u(\theta))\right\|_{0} \leq C_{i, j}\left\|\frac{\partial^{j} f}{\partial \theta^{j}}\right\|_{0}
$$

car nous pouvons permettre des pertes de rayon uniformes. Calculons quelques dérivées de $\eta_{r}$ par rapport à $\theta$ :

$$
\begin{aligned}
\frac{\partial \eta_{r}(f, u)}{\partial \theta}= & \frac{\partial f}{\partial \theta}(\cdot, u(\cdot))+f^{\prime}(\cdot, u(\cdot)) \frac{\partial u}{\partial \theta} \\
\frac{\partial^{2} \eta_{r}(f, u)}{\partial \theta^{2}}= & \frac{\partial^{2} f}{\partial \theta^{2}}(\cdot, u(\cdot))+\frac{\partial f^{\prime}}{\partial \theta}(\cdot, u(\cdot)) \frac{\partial u}{\partial \theta}+\frac{\partial f^{\prime}}{\partial \theta}(\cdot, u(\cdot)) \frac{\partial u}{\partial \theta} \\
& +f^{\prime \prime}(\cdot, u(\cdot))\left(\frac{\partial u}{\partial \theta}\right)^{2}+f^{\prime}(\cdot, u(\cdot)) \frac{\partial^{2} u}{\partial \theta^{2}} .
\end{aligned}
$$

Plus généralement nous voyons que grâce aux estimations de Cauchy (9) il nous suffit d'estimer les termes de la forme

$$
\left\|\frac{\partial^{j} f}{\partial \theta^{j}}\left(\frac{\partial u}{\partial \theta}\right)^{i_{1}}\left(\frac{\partial^{2} u}{\partial \theta^{2}}\right)^{i_{2}} \ldots\left(\frac{\partial^{n} u}{\partial \theta^{n}}\right)^{i_{n}}\right\|_{0}
$$

avec $j+i_{1}+2 i_{2}+\cdots+n i_{n}$, par rapport aux seminormes $\|f\|_{n}$ et $\|u\|_{n}$. En utilisant les inégalités de convexité de Hadamard (7) nous avons les estimations

$$
\begin{aligned}
& \left\|\frac{\partial^{j} f}{\partial \theta^{j}}\right\|_{0} \leq \tilde{C}_{0, n}^{\Gamma^{\infty}}\|f\|_{0}^{\frac{n-j}{n}}\|f\|_{n}^{\frac{j}{n}} \leq A_{n}\|f\|_{n}^{\frac{j}{n}} \\
& \left\|\frac{\partial^{s} u}{\partial \theta^{s}}\right\|_{0}^{i_{s}} \leq\left(\tilde{C}_{0, n}^{C^{\infty}}\|u\|_{0}^{\frac{n-s}{n}}\|u\|_{n}^{\frac{s}{n}}\right)^{i_{s}} \leq B_{n}\|u\|_{n^{\frac{s i s}{n}}}
\end{aligned}
$$

pour des constantes positives $A_{n}, B_{n}$ qui dépendent seulement du voisinage de $\bar{f}$ à part de $n$. En considérant un produit adéquat des inégalités ci-dessus nous avons

$$
\left\|\frac{\partial^{j} f}{\partial \theta^{j}}(\theta, u(\theta))\left(\frac{\partial u}{\partial \theta}\right)^{i_{1}}\left(\frac{\partial^{2} u}{\partial \theta^{2}}\right)^{i_{2}} \ldots\left(\frac{\partial^{n} u}{\partial \theta^{n}}\right)^{i_{n}}\right\|_{0} \leq D_{n}\|f\|_{n}^{\frac{j}{n}}\|u\|_{n^{\frac{n-j}{n}}}
$$

pour des constantes positives $D_{n}$. L'inégalité de Young implique finalement que

$$
\left\|\frac{\partial^{j} f}{\partial \theta^{j}}(\theta, u(\theta))\left(\frac{\partial u}{\partial \theta}\right)^{i_{1}}\left(\frac{\partial^{2} u}{\partial \theta^{2}}\right)^{i_{2}} \ldots\left(\frac{\partial^{n} u}{\partial \theta^{n}}\right)^{i_{n}}\right\|_{0} \leq D_{n}\left(\|f\|_{n}+\|u\|_{n}\right) .
$$

On peut en déduire donc que l'application $\eta_{r}$ est une bonne application de classe $C^{0}$.

Corolaire 6.3. - L'application $\eta_{r}$ (cf. 6. du lemme 6.2) est une bonne application de classe $C^{\infty}$ pour tout $r$ dans $(1 / 2,1]$. 
Démonstration. - Nous devons montrer que toutes les dérivées $D^{i} \eta_{r}$ sont de bonnes applications de classe $C^{0}$. Voyons ce qui se passe avec $D \eta_{r}$

$$
\begin{aligned}
D \eta_{r}(f, u)(\Delta f, \Delta u) & =\lim _{t \rightarrow 0} \frac{1}{t}[(f+t \Delta f)(\cdot, u+s \Delta u(\cdot))-f(\cdot, u(\cdot))] \\
& =f^{\prime}(\cdot, u(\cdot)) \Delta u+\Delta f(\cdot, u(\cdot)) .
\end{aligned}
$$

C'est une fonction à 4 variables dans un bon espace de Fréchet (le produit de bons espaces) qui peut s'écrire comme la composition de bonnes applications qui apparaissent dans le lemme précédent, donc c'est une bonne application. L'argument pour les dérivées d'ordre supérieur est analogue.

6.3. Les bons opérateurs $\mathbb{M}_{\alpha}, \mathbb{M}_{\alpha, \beta}$. - Nous définissons les opérateurs $\mathbb{C}$ linéaires

$$
\begin{aligned}
\mathbb{M}_{\alpha}: C^{\infty}\left(\mathbb{T}^{1}, \mathbb{C}\right)_{*} & \rightarrow C^{\infty}\left(\mathbb{T}^{1}, \mathbb{C}\right) \\
\phi & \mapsto \psi \\
\mathbb{M}_{\alpha, \beta}: C^{\infty}\left(\mathbb{T}^{1}, \mathbb{C}\right) & \rightarrow C^{\infty}\left(\mathbb{T}^{1}, \mathbb{C}\right) \\
\tilde{\phi} & \mapsto \tilde{\psi}
\end{aligned}
$$

où $C^{\infty}\left(\mathbb{T}^{1}, \mathbb{C}\right)_{*}$ est l'espace des fonctions dans $C^{\infty}\left(\mathbb{T}^{1}, \mathbb{C}\right)$ de moyenne nulle et $\psi(\tilde{\psi})$ est la solution de l'équation cohomologique (1) (de l'équation cohomologiquement tordue (3)) dont le membre droit est $\phi(\tilde{\phi})$. Ainsi définis ces opérateurs sont inversibles (compte tenue de la normalisation $\int_{\mathbb{T}^{1}} \psi d \theta=0$ ).

Lemma 6.4. - Les opérateurs $\mathbb{M}_{\alpha}, \mathbb{M}_{\alpha, \beta}$ sont des bonnes applications au sens de Hamilton.

Démonstration. - La théorie classique des séries de Fourier d'une fonction $f$ dans $C^{\infty}\left(\mathbb{T}^{1}, \mathbb{C}\right)$ assure que les coefficients de sa série de Fourier vérifient les inégalités

$$
\begin{aligned}
\|f\|_{i} & \leq B_{i} \sup _{k \in \mathbb{Z}}\left((1+|k|)^{i+2}|\hat{f}(k)|\right) \\
\sup _{k \in \mathbb{Z}}\left((1+|k|)^{i}|\hat{f}(k)|\right) & \leq C_{i}\|f\|_{i}
\end{aligned}
$$

pour des constantes positives $B_{i}, C_{i}$ qui dépendent seulement de $i$ dans $\mathbb{N}$. Soit $\phi$ dans $C^{\infty}\left(\mathbb{T}^{1}, \mathbb{C}\right)$. Les inégalités ci-dessus et la condition diophantienne sur $\alpha$ impliquent que pour tout $i \geq 0$ il existe des constantes positives $C_{i}^{\prime}, B_{i+2}^{\prime}$ telles que

$$
\begin{aligned}
\left\|\mathbb{M}_{\alpha}(\phi)\right\|_{i} & \leq C_{i} \sup _{k \in \mathbb{Z}}\left((1+|k|)^{i+2}\left|\frac{\hat{\phi}(k)}{e^{2 \pi i k \alpha}-1}\right|\right) \\
& \leq C_{i}^{\prime} \sup _{k \in \mathbb{Z}}\left((1+|k|)^{i+4}|\hat{\phi}(k)|\right) \\
& \leq B_{i+2}^{\prime}\|v\|_{i+2} .
\end{aligned}
$$


La preuve pour $\mathbb{M}_{\alpha, \beta}$ étant analogue, nous l'omettons.

6.4. Correction du nombre de rotation transversal. - Pour toute $f$ dans $\Gamma^{\infty}\left(\mathbb{T}^{1}, \Omega(\overline{\mathbb{D}})\right)$ telle que la taille $C^{0}$ de $f-\lambda z$ est suffisamment petite et pour toute courbe continue $u: \mathbb{T}^{1} \rightarrow \mathbb{D}$ de taille $C^{0}$ petite nous pouvons définir uniformément la fonction $\log f^{\prime}(\theta, u(\theta))$ qui vérifie $\log \lambda=2 \pi i \beta$, car le degré de la fonction $f^{\prime}(\theta, u(\theta))$ dépend continûment de la paire $(f, u)$. Dans ce cas nous pouvons calculer l'intégrale suivante

$$
I(f, u)=\frac{1}{2 \pi i} \int \log f^{\prime}(\theta, u(\theta)) d \theta .
$$

Notons que quand la courbe $u$ est invariante par la dynamique associée à $f$, et de degré nul, ce nombre coïncide avec le nombre de rotation transversal $\varrho_{t r}(u)$. Avec cette définition nous pouvons donner un sens à l'affirmation suivante « pour n'importe quelle paire $(f, u)$ il existe toujours un nombre complexe $t$ qui corrige le nombre de rotation transversal pour lui donner la valeur $\beta$ ».

Proposition 6.5. - Pour tout nombre réel $\beta$ dans $[0,1)$, pour toute application $f$ dans un voisinage $U$ de $f_{0} \equiv e^{2 \pi i \beta} z$ dans $\Gamma^{\infty}\left(\mathbb{T}^{1}, \Omega(\overline{\mathbb{D}})\right)$ et pour toute courbe $u$ dans une voisinage $V$ de $u_{0} \equiv 0$ dans $C^{\infty}\left(\mathbb{T}^{1}, \mathbb{C}\right)$, la fonction $t: U \times V \rightarrow \mathbb{C}$ définie par l'égalité $\mathscr{I}\left(e^{t} f, u\right)=\beta$ est une bonne application de classe $C^{\infty}$. En plus on a

$$
|t| \leq\left\|f^{\prime}-e^{2 \pi i \beta}\right\|_{C^{0}}
$$

Démonstration. - En fait, la formule (12) nous permet de calculer explicitement la valeur de $t$. Plus précisément, soient $U, V$ les voisinages qui nous permettent de calculer l'intégrale $I$ comme au paragraphe précédent, alors

$$
\begin{aligned}
I\left(e^{t} f, u\right) & =\frac{1}{2 \pi i} \int \log e^{t} f^{\prime}(\theta, u(\theta)) d \theta \\
\beta & =\frac{1}{2 \pi i}\left(t+\int \log f^{\prime}(\theta, u(\theta)) d \theta\right) .
\end{aligned}
$$

Ainsi $t$ est une bonne application de classe $C^{\infty}$ d'après la proposition 5.1 et les lemmes 6.2, 6.3. L'estimation (13) s'obtient facilement à l'aide de (14).

Dorénavant chaque fois que nous écrivons $e^{t} f(\theta, u(\theta))$, et qu'il n'y a pas lieu à confusion, il faudra penser toujours que $t=t(f, u)$.

TOME $138-2010-\mathrm{N}^{\circ} 2$ 
6.5. La bonne application $u_{1}$. - L'égalité $\mathscr{I}\left(e^{t} f, u\right)=\beta$ et l'hypothèse $\alpha$ dans $\mathbb{C D}$ nous permettent de résoudre l'équation

$$
e^{t} f^{\prime}(\theta, u(\theta))=e^{2 \pi i \beta} \frac{u_{1}(\theta+\alpha)}{u_{1}(\theta)}
$$

avec $u_{1} \in C^{\infty}\left(\mathbb{T}^{1}, \mathbb{C}\right)_{0}$. En fait, on montre le

LEMMA 6.6. - L'application $(f, u) \mapsto u_{1}$ est une bonne application au sens de Hamilton.

Démonstration. - En effet, dans les bons voisinages, où la fonction $t$ est bien définie, l'équation (15) se réduit à l'équation cohomologique

$$
t+\log f^{\prime}(\theta, u(\theta))=2 \pi i \beta+\tilde{u}_{1}(\theta+\alpha)-\tilde{u}_{1}(\theta) .
$$

On voit que la condition $I\left(e^{t} f, u\right)=\beta$ est la condition intégrale nécessaire pour résoudre cette équation. Pour assouplir la notation nous introduisons la fonction $l(f, u)(\theta)=\log f^{\prime}(\theta, u(\theta))$. Il est clair que l'application $(f, u) \mapsto l(f, u)$ est une bonne application dans les voisinages que nous considérons ici. Nous avons alors

$$
\tilde{u_{1}}(\theta)=\mathbb{M}_{\alpha}(l(u, f))(\theta) .
$$

L'application $(f, u) \mapsto \tilde{u_{1}}$ est une bonne application au sens de Hamilton d'après les résultats de la section 6.3, et il en est de même de l'application

$$
(f, u) \longmapsto u_{1}=e^{\tilde{u}_{1}} .
$$

$\mathrm{Si}$, en outre, les voisinages $V, U$ sont suffisamment petits, la distance $\left|u_{1}(\theta)-1\right|$ est uniformément bornée, et en particulier $u_{1}(\theta)$ ne s'annule pas. Il est immédiat de vérifier que $u_{1}$ satisfait l'équation (15).

6.6. Le théorème 4.2 sous la forme d'un problème de fonction implicite. - Considérons l'application $\Theta:\left(U \subset \Gamma^{\infty}\left(\mathbb{T}^{1}, \Omega(\overline{\mathbb{D}})\right)\right) \times\left(V \subset C^{\infty}\left(\mathbb{T}^{1}, \mathbb{C}\right)\right) \rightarrow$ $C^{\infty}\left(\mathbb{T}^{1}, \mathbb{C}\right)$ définie par

$$
\Theta(f, u)=e^{t} f(\theta, u(\theta))-u(\theta+\alpha)
$$

où l'application $t(f, u)$ et les ensembles $U, V$ sont définis dans la proposition 6.5. Cette application est une bonne application de classe $C^{\infty}$. On peut sans peine voir que le fait que $u$ soit invariante pour la dynamique holomorphe fibrée associée à $e^{t} f$ est équivalente au fait que $\Theta(f, u)$ soit identiquement nulle. Notons que dans le cas où $f_{0} \equiv \lambda z$ et $u_{0} \equiv 0$ nous avons

$$
\Theta\left(f_{0}, u_{0}\right)=0 \text {. }
$$

La preuve du théorème 4.2 sera une application directe du théorème des fonctions implicites de Hamilton, c'est-à-dire, la courbe invariante $u$ sera définie 
d'une façon implicite à partir de l'équation $\Theta(f, u)=0$ autour de la solution déjà connue $\left(f_{0}, u_{0}\right)$.

6.6.1. Inversion de la différentielle. — La partie la plus importante pour appliquer le théorème de Hamilton est l'inversion de la différentielle par rapport à la deuxième variable, ce qui dans notre cas se traduit par trouver une bonne application continue $L(f, u, \Delta g)$, linéaire en $\Delta g$, définie pour toute paire $(f, u)$ dans un voisinage de $\left(f_{0}, u_{0}\right)$ et pour tout $\Delta g$ dans $C^{\infty}\left(\mathbb{T}^{1}, \mathbb{C}\right)$ de façon que si nous posons $\Delta u=L(f, u, \Delta g)$ nous avons

$$
D_{2} \Theta(f, u) \Delta u=\Delta g .
$$

Dans ce qui se suit nous allons résoudre cette équation (en $\Delta u$ dans $C^{\infty}\left(\mathbb{T}^{1}, \mathbb{C}\right)$ ) d'une façon formelle. La différentielle partielle de $\Theta$ par rapport à $u$ en la direction $\Delta u$ est

$$
D_{2} \Theta(f, u) \Delta u=e^{t} f(\cdot, u(\cdot))\left(\partial_{u} t \cdot \Delta u\right)+e^{t} f^{\prime}(\cdot, u(\cdot)) \Delta u-\Delta u(\cdot+\alpha)
$$

où $\partial_{u} t \cdot \Delta u$ est la différentielle de $t$ par rapport à $u$ dans la direction $\Delta u$, qui est un nombre complexe. Nous prenons $u_{1}(f, u)=u_{1}$ comme dans (15). L'équation (17) devient donc

$$
e^{t} f(\cdot, u)\left(\partial_{u} t \cdot \Delta u\right)+\frac{u_{1}(\cdot+\alpha)}{u_{1}} e^{2 \pi i \beta} \Delta u-\Delta u(\cdot+\alpha)=\Delta g
$$

Avec la notation $\widetilde{\Delta u}=\frac{\Delta u}{u_{1}}, \widetilde{\Delta g}=\frac{\Delta g}{u_{1}(\cdot+\alpha)}, \tilde{f}=\frac{e^{t} f(\cdot, u)}{u_{1}(\cdot+\alpha)}$ nous avons

$$
e^{2 \pi i \beta} \widetilde{\Delta u}(\theta)-\widetilde{\Delta u}(\theta+\alpha)=\widetilde{\Delta g}(\theta)-\left(\partial_{u} t \cdot \Delta u\right) \tilde{f}(\theta) .
$$

En appliquant l'opérateur $\mathbb{C}$-linéaire (inversible) $\mathbb{M}_{\alpha, \beta}$ aux deux cotés on obtient de façon équivalente

$$
\widetilde{\Delta u}=\mathbb{M}_{\alpha, \beta}(\widetilde{\Delta g})-\left(\partial_{u} t \cdot \Delta u\right) \mathbb{M}_{\alpha, \beta}(\tilde{f}) .
$$

On peut calculer explicitement la valeur de $\partial_{u} t \cdot \Delta u$, car en dérivant l'égalité $I\left(e^{t} f, u\right)=\beta(\operatorname{voir}(14))$ dans la direction $\Delta u$ nous obtenons

$$
\frac{1}{2 \pi i}\left(\left(\partial_{u} t \cdot \Delta u\right)+\int_{\mathbb{T}^{1}} \frac{f^{\prime \prime}(\theta, u(\theta)) \Delta u(\theta)}{f^{\prime}(\theta, u(\theta))} d \theta\right)=0
$$

TOME $138-2010-\mathrm{N}^{\mathrm{O}} 2$ 
ce qui ajouté à l'égalité (18) nous donne

$$
\begin{aligned}
\partial_{u} t \cdot \Delta u & =-\int_{\mathbb{T}^{1}} \frac{f^{\prime \prime}(\theta, u(\theta)) \Delta u(\theta)}{f^{\prime}(\theta, u(\theta))} d \theta \\
& =-\int_{\mathbb{T}^{1}} \frac{f^{\prime \prime}(\theta, u(\theta)) u_{1}(\theta)\left(\mathbb{M}_{\alpha, \beta}(\widetilde{\Delta g})(\theta)-\left(\partial_{u} t \Delta u\right) \mathbb{M}_{\alpha, \beta}(\tilde{f})(\theta)\right)}{f^{\prime}(\theta, u(\theta))} d \theta \\
\partial_{u} t \cdot \Delta u & =\frac{-\int_{\mathbb{T}^{1}} \frac{f^{\prime \prime}(\theta, u(\theta)) u_{1}(\theta)}{f^{\prime}(\theta, u(\theta))} \mathbb{M}_{\alpha, \beta}(\widetilde{\Delta g})(\theta) d \theta}{1-\int_{\mathbb{T}^{1}} \frac{f^{\prime \prime}(\theta, u(\theta)) u_{1}(\theta)}{f^{\prime}(\theta, u(\theta))} \mathbb{M}_{\alpha, \beta}(\tilde{f})(\theta) d \theta} .
\end{aligned}
$$

Nous posons finalement

$$
\Delta u=u_{1}\left(\mathbb{M}_{\alpha, \beta}(\widetilde{\Delta g})-\left(E \mathbb{M}_{\alpha \beta}(\tilde{f})\right)\right.
$$

où $E=\partial_{u} t \cdot \Delta u$ comme dans (19). On voit que si $f^{\prime \prime}(\theta, u(\theta))$ est suffisamment petit par rapport à la taille $C^{0}$ de $u_{1}$ et $\mathbb{M}_{\alpha, \beta}(\tilde{f})$, le nombre complexe $\partial_{u} t \cdot \Delta u$ est bien défini et en plus l'application $(f, u, \Delta g) \mapsto \partial_{u} t \cdot \Delta u$ est une bonne application au sens de Hamilton. Il est direct aussi que la définition ci-dessus pour $\Delta u$ vérifie l'équation (17), et l'application $(u, f, \Delta g) \mapsto \Delta u$ est une bonne application au sens de Hamilton dans un voisinage adéquat de la paire $\left(f_{0}, u_{0}\right)$. Or, les conditions sur $f^{\prime \prime}, u_{1}, M_{\alpha, \beta}(\tilde{f})$ s'obtiennent en rétrécissant suffisamment le voisinage autour de $\left(f_{0}, u_{0}\right)$ qui sert à calculer $t$. Nous avons en main tous les ingrédients pour appliquer le théorème de Hamilton à l'application $\Theta$ : Il existe une bonne application de classe $C^{\infty}$

$$
\begin{aligned}
\Xi: \widetilde{U} \subset \Gamma^{\infty}\left(\mathbb{T}^{1}, \Omega(\overline{\mathbb{D}})\right) & \longrightarrow \mathbb{C} \times C^{\infty}\left(\mathbb{T}^{1}, \mathbb{C}\right) \\
\Xi(f) & \longmapsto(t, u)
\end{aligned}
$$

définie dans un voisinage $\tilde{U}$ de $f_{0}$ (une $C^{r}$ boule, pour un certain $r \in \mathbb{N}$ ) telle que la courbe $u$ est invariante pour la dynamique holomorphe fibrée associée à $e^{t} f$, de degré nul et de nombre transversal de rotation égal à $\beta$, ce qui donne une version plus précise du théorème 4.2. Soient $\varepsilon>0$ et $r \in \mathbb{N}$. Nous posons

$$
B_{\bar{\varepsilon}}^{r}=\left\{f \in \Gamma^{\infty}\left(\mathbb{T}^{1}, \Omega(\overline{\mathbb{D}})\right) \mid\|f-\lambda z\|_{r}<\bar{\varepsilon}\right\} .
$$

ThÉORÈme 6.1 . - Pour toute paire $(\alpha, \beta)$ qui vérifie l'hypothèse $\mathbb{C D}_{1}$ il existe $\bar{\varepsilon}>0$, un nombre naturel $r \geq 2$ et une bonne application de classe $C^{\infty}$ au sens de Hamilton, $\Xi: B_{\bar{\varepsilon}}^{r} \subset \Gamma^{\infty}\left(\mathbb{T}^{1}, \Omega(\overline{\mathbb{D}})\right) \rightarrow \mathbb{C} \times C^{\infty}\left(\mathbb{T}^{1}, \mathbb{C}\right)$ tels que

- $\Xi(\lambda z)=(0,0)$.

- Si on écrit $\Xi(f)=(t, u)$ alors la courbe u est invariante par la dynamique holomorphe fibrée $F^{*}(\theta, z)=\left(\theta+\alpha, e^{t} f(\theta, z)\right)$, est de degré nul et son nombre de rotation transversal est $\varrho_{t r}\left(e^{t} f, u\right)=\beta$. 
- La projection sur la première coordonnée $\pi_{1} \Xi(f)$ coïncide avec $t\left(f, \pi_{2} \Xi(f)\right)$ où $\pi_{2} \Xi(f)$ est la projection sur la deuxième coordonnée et $t$ est la fonction définie dans la proposition 6.5. La paire $(t, u)$ est uniquement déterminée par ces propriétés, pour $u$ dans un voisinage de $u_{0} \equiv 0$.

\section{Preuve du théorème 4.1, un argument de transversalité}

Soit $\left\{f_{s}\right\}_{s \in \Sigma \subset \mathbb{C}}$ une famille lisse à un paramètre complexe de fonctions dans $\Gamma^{\infty}\left(\mathbb{T}^{1}, \Omega(\overline{\mathbb{D}})\right)$. Si la famille $\left\{f_{s}\right\}$ vérifie aussi que $\left\|f_{s}-\lambda z\right\|_{r}<\bar{\varepsilon}$ pour tout $s$ dans un certain disque $D(0, R) \subset \Sigma \subset \mathbb{C}$, où $\bar{\varepsilon}, r$ sont ceux du théorème 6.1, nous pouvons définir une application $t: D(0, R) \rightarrow \mathbb{C}$ de classe $C^{\infty}$, et une application $u: D(0, R) \rightarrow C^{\infty}\left(\mathbb{T}^{1}, \mathbb{C}\right)$ de classe $C^{\infty}$ par

$$
\begin{aligned}
& s \mapsto t(s)=\pi_{1} \Xi\left(f_{s}\right) \\
& s \mapsto u(s)=\pi_{2} \Xi\left(f_{s}\right)
\end{aligned}
$$

où l'application $\Xi$ est celle fournie par le théorème 6.1. Dans ce cas la courbe $u(s)$ est invariante par la dynamique holomorphe fibrée $F_{s}(\theta, z)=$ $\left(\theta+\alpha, e^{t(s)} f_{s}(\theta, z)\right)$, est de degré nul et son nombre de rotation transversal est $\varrho_{t r}(u(s))=\beta$. Le but de cette section est de montrer que sous l'hypothèse de transversalité de la famille $\left\{f_{s}\right\}_{s \in \Sigma}$ on peut choisir un rayon $R>0$ et trouver un paramètre $s^{*}$ dans $D(0, R)$ tel que $t\left(s^{*}\right)=0$, c'est-à-dire, qu'on n'a pas besoin de faire une correction $e^{t}$ sur la dynamique $F_{s^{*}}(\theta, z)=\left(\theta+\alpha, f_{s^{*}}(\theta, z)\right)$ afin d'obtenir la courbe invariante avec le bon nombre transversal de rotation.

Le lecteur aura remarqué qu'un théorème classique des fonctions implicites semblerait pouvoir s'appliquer à l'égalité

$$
t+\int_{\mathbb{T}^{1}} \log f_{s}^{\prime}\left(\theta, u_{s}(\theta)\right) d \theta=2 \pi i \beta
$$

afin d'exprimer $s$ en fonction de $t$ autour de la paire $(0, t(0))$. C'est bien le cas, mais on aura a faire des estimations fines pour montrer que $t=0$ se trouve sur le domaine de la fonction implicite $s(t)$. Nous avons choisi un argument à la Rouché car ceci nous semble plus transparent.

7.0.1.1. L'Indice de Kronecker. - Nous allons faire dans cette section un petit rappel sur l'indice de Kronecker d'une fonction du plan $\mathbb{R}^{2}$ vers $\mathbb{R}^{2}$ par rapport à un disque. Soit $G: D \subset \mathbb{R}^{2} \rightarrow \mathbb{R}^{2}$ une fonction de classe $C^{2}$, qui s'écrit $G(x)=\left(g_{1}(x), g_{2}(x)\right)$ définie sur un disque $D$ du plan. Nous définissons l'indice de Kronecker de $G$ sur le bord $\partial D$ par l'intégrale

$$
n(G ; D)=\frac{1}{2 \pi} \int_{\partial D} \frac{g_{1} d g_{2}-g_{2} d g_{1}}{g_{1}^{2}+g_{2}^{2}}
$$

TOME $138-2010-\mathrm{N}^{\mathrm{O}} 2$ 
quand il n'y a pas de zéros de $G$ sur le bord $\partial D$. Ce nombre mesure le nombre de tours que la courbe $G(\partial D)$ fait autour de zéro. En fait, il n'est pas difficile de voir que

$$
n(G ; D)=\frac{1}{2 \pi} \int_{\partial D} G^{*}(\delta \theta)=\frac{1}{2 \pi} \int_{G \circ \partial D} \delta \theta,
$$

où $\delta \theta$ est la 1-forme d'élément d'angle autour de l'origine complexe. Parmi les diverses propriétés de l'indice de Kronecker nous allons utiliser seulement celles qui sont contenues dans la proposition suivante, dont la preuve se trouve dans [21] :

Proposition 7.1. - Soit $G: D \subset \mathbb{R}^{2} \rightarrow \mathbb{R}^{2}$ et $J: D \subset \mathbb{R}^{2} \rightarrow \mathbb{R}^{2}$ deux fonctions de classe $C^{2}$. On a

1. Si 0 n'appartient pas au segment $[G(z), J(z)]$ pour tout $z$ dans $\partial D$ alors $n(G ; D)=n(J ; D)$.

2. Si $G$ n'a pas de zéros dans le disque $D$ alors $n(G ; D)=0$.

3. Si $|G(z)|<|J(z)|$ et $J(z) \neq 0$ pour tout $z$ dans $\partial D$ alors $n(J+G ; D)=$ $n(J ; D)$.

4. Si $G$ a un zéro unique et non dégénéré dans $D$ alors $n(G ; D)= \pm 1$.

On dit qu'un point $z$ dans $D$ est un zéro non dégénéré de $G$ si le déterminant de la matrice jacobienne $J(G)$ de $G$ est non nul. Il est clair que 2.,3. sont une conséquence de 1 . Nous allons utiliser cette proposition pour montrer l'existence d'un zéro pour une fonction qui n'est pas très bien comprise, en la comparant à une autre qui possède un zéro unique et non dégénéré, dont l'indice de Kronecker n'est pas nul.

7.1. La fonction $t(s)$. - Nous allons étudier par la suite le comportement de la fonction $t(s)$. Nous savons d'après (14) qu'on a explicitement

$$
t(s)=2 \pi i \beta-\int_{\mathbb{T}^{1}} \log f_{s}^{\prime}(\theta, u(s)(\theta)) d \theta
$$

d'où on peut calculer la différentielle de $t$ au point $s$ dans la direction $\Delta s$ par

$$
D t(s) \Delta s=-\int_{\mathbb{T}^{1}} \frac{\partial_{s} f_{s}^{\prime}(\theta, u(s)(\theta)) \Delta s+f_{s}^{\prime \prime}(\theta, u(s)(\theta)) D u(s) \Delta s}{f_{s}^{\prime}(\theta, u(s)(\theta))} d \theta .
$$

Le théorème des fonctions implicites de Hamilton nous fournit aussi l'expression de la différentielle de la fonction implicite engendrée, ce qui nous permet de calculer

$$
\begin{aligned}
D u(s) \Delta s & =D \pi_{2} \Xi\left(f_{s}\right)\left[\partial_{s} f_{s} \Delta s\right] \\
& =-\left(D_{2} \Theta\left(f_{s}, \pi_{2} \Xi\left(f_{s}\right)\right)\right)^{-1}\left[D_{1} \Theta\left(f_{s}, \pi_{2} \Xi\left(f_{s}\right)\right)\left[\partial_{s} f_{s} \Delta s\right] .\right.
\end{aligned}
$$

Cette égalité nous permet d'énoncer et montrer le 
Lemma 7.2. - S'il existe une constante $T>1$ telle que $\left\|\partial_{s} f_{s}\right\|_{0}<T$ pour tout $s$ dans $D(0, R)$ alors il existe une constante positive $T^{\prime}$, qui dépend seulement de la paire $(\alpha, \beta)$ et de $T$, telle que

$$
\|D u(s) \Delta s\|_{0}<T^{\prime}|\Delta s|
$$

Nous écrivons $D t(s)=w(s)+A(s)$ avec $w(s)$ une matrice appartenant à $\mathbb{M}_{2}(\mathbb{R})$ et la matrice $A(s) \in \mathbb{M}_{2}(\mathbb{R})$ de taille contrôlée par la taille de $f_{s}^{\prime \prime}$. Plus précisément

$$
\begin{aligned}
& w(s)=-\int_{\mathbb{T}^{1}}\left(f_{s}^{\prime}(\theta, u(s)(\theta))\right)^{-1} \partial_{s} f_{s}^{\prime}(\theta, u(s)(\theta)) d \theta \\
& A(s)=\int_{\mathbb{T}^{1}}\left(f_{s}^{\prime}(\theta, u(s)(\theta))\right)^{-1} f_{s}^{\prime \prime}(\theta, u(s)(\theta)) D u(s) d \theta .
\end{aligned}
$$

Si on suppose qu'il existe des nombres réels positifs $\varepsilon_{0}, \varepsilon_{1}, \varepsilon_{2}$ tels que $\left\|f_{s}\right\|_{r} \leq \varepsilon_{0}$, $\left\|f_{s}^{\prime}-\lambda\right\|_{r} \leq 10 \varepsilon_{1},\left\|f_{s}^{\prime \prime}\right\|_{r} \leq \varepsilon_{2}$ et $\varepsilon_{0}+\varepsilon_{1}+\varepsilon_{2} \leq \bar{\varepsilon}$ alors on a que

$$
\|A(s)\|_{\mathfrak{L}}<2 T^{\prime} \varepsilon_{2} \text {. }
$$

Nous pouvons contrôler aussi la distance $\|w(s)-w(0)\|_{\mathfrak{L}}$ sur le bord d'un disque $D(0, R)$. Pour cela nous montrons le

LEMmA 7.3. - S'il existe une constante $T \geq 0$ telle que $\left\|\partial_{s}^{2} f_{s}^{\prime}\right\|_{0} \leq T$ et $\left\|\partial_{s} f_{s}^{\prime \prime}\right\|_{0} \leq T$ alors on $a$

$$
\left\|\partial_{s} f_{s}^{\prime}(\theta, z)-\left.\partial_{s} f_{s}^{\prime}\right|_{s=0}(\theta, z)\right\|_{\mathfrak{L}} \leq\left\|\partial_{s}^{2} f_{s}^{\prime}\right\|_{0}|s| \leq T R
$$

et

$$
\left.|| \partial_{s} f_{s}^{\prime}\right|_{s=0}(\theta, u(0)(\theta))-\left.\left.\partial_{s} f_{s}^{\prime}\right|_{s=0}(\theta, 0)\left\|_{\mathfrak{L}} \leq\right\| \partial_{s} f_{s}^{\prime \prime}\right|_{s=0}\left\|_{0}\right\| u(s) \|_{0} \leq T T^{\prime} R .
$$

Il existe donc une constante positive $T^{\prime \prime}$, qui dépend seulement de $T$ et $(\alpha, \beta)$, telle que

$$
\|w(s)-w(0)\|_{\mathfrak{L}} \leq T^{\prime \prime} R .
$$

7.2. Transversalité et fin de la preuve. - Afin de trouver un zéro simple de $t(s)$ nous cherchons à utiliser le point 3 . de la proposition 7.1. Considérons la fonction affine $W(s)=t(0)+w(0) s$. L'hypothèse de transversalité

$$
\left[\left.\partial_{s} \int_{\mathbb{T}^{1}} f_{s}^{\prime}(\theta, 0) d \theta\right|_{s=0}\right]_{\mathfrak{L}}>L^{-1},
$$

pour une constante $L>1$, impliquera que $W$ croît suffisamment vite, ce qui va nous permettre de la comparer à la différence $t(s)-W(s)$ sur le bord d'un disque $D(0, R)$, de rayon $R$ assez grand de façon que le zéro (unique et non dégénéré)

$$
\tilde{s}=-w(0)^{-1} t(0)
$$

TOME $138-2010-\mathrm{N}^{\mathrm{O}} 2$ 
de $W$, soit contenu dans ce disque. Si on demande que $\left\|f_{s=0}^{\prime}-\lambda\right\| \leq \varepsilon_{1}$, le lemme 4.1 et l'estimation (13) nous donnent $|\tilde{s}| \leq L \varepsilon_{1}$. D'après (20) et (21) la différence entre les fonctions $W$ et $t$ sur le bord du disque $D(0, R)$ est bornée par

$$
\begin{aligned}
|W(s)-t(s)| & \leq R\|D(W-t)\|_{\mathfrak{L}} \\
& \leq R\left(\|w(s)-w(0)\|_{\mathfrak{L}}+\|A(s)\|_{\mathfrak{L}}\right) \\
& \leq R\left(T^{\prime \prime} R+2 T^{\prime} \varepsilon_{2}\right) .
\end{aligned}
$$

D'autre part la taille de $W$ est minorée sur le bord du même disque par

$$
|W(s)|>R L^{-1}-\varepsilon_{1} .
$$

LEMMA 7.4. - Si $R=2 L \varepsilon_{1}$ et $\bar{\varepsilon}$ est suffisamment petit alors il existe un zéro $s^{*}$ de $t$ dans $D(0, R)$.

Démonstration. - Avec ce choix pour $R$ on a

$$
R L^{-1}-\varepsilon_{1}>T^{\prime \prime} R^{2}+2 T^{\prime} \varepsilon_{2} R
$$

Ceci nous permet d'appliquer le point 3. de la proposition 7.1 aux fonctions $W$ et $t$, et assurer la présence d'un zéro $s^{*}$ de $t$ dans $D(0, R)$. Or, il n'y a que deux possibilités, soit $t$ s'annule sur le bord du disque, ou bien l'indice $n(t, D(0, R))=n(W, D(0, R))= \pm 1$ et le point 2 . de la proposition 7.1 nous donne l'existence de $s^{*}$.

Nous venons de montrer ainsi la

Proposition 7.5. - Pour toute paire $(\alpha, \beta)$ qui vérifie l'hypothèse $\mathbb{C D}_{1}$ et pour toutes constantes $L>1, T>1$ il existe $\bar{\varepsilon}>0$, un nombre naturel $r \geq$ 2 , qui dépend seulement de la paire $(\alpha, \beta)$, tels que si une famille lisse à un paramètre complexe $\left\{f_{s}\right\}_{s \in \Sigma \subset \mathbb{C}}$ de fonctions de $\mathbb{T}^{1}$ vers $\Gamma^{\infty}\left(\mathbb{T}^{1}, \Omega(\overline{\mathbb{D}})\right)$ vérifie pour des nombres réels positifs $\varepsilon_{0}, \varepsilon_{1}, \varepsilon_{2}, \varepsilon_{0}+10 \varepsilon_{1}+\varepsilon_{2} \in(0, \bar{\varepsilon}]$,

- $\left[\left.\partial_{s} \int_{\mathbb{T}^{1}} f_{s}^{\prime}(\theta, 0) d \theta\right|_{s=0}\right]_{\mathfrak{L}}>L^{-1} ;\left\|f_{s=0}^{\prime}-\lambda\right\|_{r} \leq \varepsilon_{1}$

- $\left\|f_{s}\right\|_{r} \leq \varepsilon_{0},\left\|f_{s}^{\prime}-\lambda\right\|_{r} \leq 10 \varepsilon_{1},\left\|f_{s}^{\prime \prime}\right\|_{r} \leq \varepsilon_{2}$ pour tout $s$ dans $D\left(0,2 L \varepsilon_{1}\right) \subset \Sigma$

- $\left\|\partial_{s}^{2} f_{s}^{\prime}\right\|_{0}+\left\|\partial_{s} f_{s}^{\prime \prime}\right\|_{0} \leq T$ pour tout $s$ dans $D\left(0,2 L \varepsilon_{1}\right) \subset \Sigma$

alors il existe un paramètre $s^{*}$ dans le disque $D\left(0,2 L \varepsilon_{1}\right) \subset \Sigma$ et une courbe $u: \mathbb{T}^{1} \rightarrow \mathbb{D}$ de classe $C^{\infty}$ qui est invariante par la dynamique holomorphe fibrée $F_{s^{*}}(\theta, z)=\left(\theta+\alpha, f_{s^{*}}(\theta, z)\right)$, est de degré nul et son nombre de rotation transversal est $\varrho_{t r}\left(f_{s^{*}}, u\right)=\beta$. 
Nous pouvons finir à présent la preuve du théorème 4.1 en montrant qu'on peut se ramener aux hypothèses de la proposition ci-dessus. Supposons qu'il existe $\tilde{\varepsilon}>0$ et une constante $T>1$ tels que

$$
\begin{aligned}
\left\|\rho_{0, s}\right\|_{r} & \leq 10 \tilde{\varepsilon} \\
\left\|\rho_{1, s}\right\|_{r} & \leq 10 \tilde{\varepsilon} \quad, \quad\left\|\rho_{1, s=0}\right\|_{r} \leq \tilde{\varepsilon} \\
\left\|\rho_{s}\right\|_{r} & \leq T .
\end{aligned}
$$

Nous faisons un changement d'échelle sur $z$ de taille $m>1$, c'est-à-dire, nous définissons une nouvelle famille lisse $\left\{\tilde{f}_{s}\right\}_{s \in \Sigma \subset \mathbb{C}}$ par

$$
\tilde{f}_{s}(\theta, z)=m f_{s}\left(\theta, m^{-1} z\right)
$$

ce qui avec la notation usuelle nous donne

$$
\begin{aligned}
\left\|\tilde{\rho}_{0, s}\right\|_{r} & \leq m 10 \tilde{\varepsilon} \\
\left\|\tilde{\rho}_{1, s}\right\|_{r} & \leq 10 \tilde{\varepsilon} \quad, \quad\left\|\rho_{1, s=0}\right\|_{r} \leq \tilde{\varepsilon} \\
\left\|\tilde{\rho}_{s}\right\|_{r} & \leq m^{-1} T .
\end{aligned}
$$

Si nous posons $m=3 T \bar{\varepsilon}^{-1}$ (quitte à diminuer $\bar{\varepsilon}$ on a que $m>1$ ) et $\tilde{\varepsilon} \leq \frac{\bar{\varepsilon}^{2}}{9 T}$ nous avons

$$
\begin{aligned}
\left\|\tilde{\rho}_{0, s}\right\|_{r} & \leq \frac{\bar{\varepsilon}}{3} \\
\left\|\tilde{\rho}_{1, s}\right\|_{r} & \leq 10 \tilde{\varepsilon}<\frac{\bar{\varepsilon}}{3} \quad, \quad\left\|\rho_{1, s=0}\right\|_{r} \leq \tilde{\varepsilon} \\
\left\|\tilde{\rho}_{s}\right\|_{r} & \leq \frac{\bar{\varepsilon}}{3} .
\end{aligned}
$$

La proposition 7.5 nous donne donc un paramètre $s^{*}$ dans le disque $D(0,2 L \tilde{\varepsilon})$ et une courbe $u$ de classe $C^{\infty}$ qui est invariante par la dynamique holomorphe fibrée $\tilde{F}_{s^{*}}$, ce qui signifie

$$
\begin{aligned}
\tilde{F}_{s^{*}}(\theta, u(\theta)) & =(\theta+\alpha, u(\theta+\alpha)) \\
\left(\theta+\alpha, m f_{s^{*}}\left(\theta, m^{-1} u(\theta)\right)\right) & =(\theta+\alpha, u(\theta+\alpha)) \\
f_{s^{*}}\left(\theta, m^{-1} u(\theta)\right) & =m^{-1} u(\theta+\alpha) .
\end{aligned}
$$

On voit ainsi que la courbe $m^{-1} u$ est invariante par la dynamique holomorphe fibrée $F_{s^{*}}$. En plus $\tilde{f}_{s}^{\prime}(\theta, z)=f_{s}^{\prime}\left(\theta, m^{-1} z\right)$, donc le nombre de rotation transversal de la courbe $m^{-1} u$ est aussi égal à $\beta$ ce qui termine la preuve du théorème 4.1

TOME $138-2010-\mathrm{N}^{\mathrm{O}} 2$ 


\section{BIBLIOGRAPHIE}

[1] A. Avila \& R. KRIKORIAN - « Reducibility or nonuniform hyperbolicity for quasiperiodic Schrödinger cocycles », Ann. of Math. 164 (2006), p. 911940.

[2] J.-B. Bost - «Tores invariants des systèmes dynamiques hamiltoniens (d'après Kolmogorov, Arnol'd, Moser, Rüssmann, Zehnder, Herman, Pöschel,...) », Séminaire Bourbaki, vol. 1984/85, Astérisque 133-134 (1986), p. $113-157$.

[3] J. Bourgain - «On Melnikov's persistency problem », Math. Res. Lett. 4 (1997), p. 445-458.

[4] L. Carleson \& T. W. Gamelin - Complex dynamics, Universitext, Springer, 1993.

[5] J. W. S. CASsELS - An introduction to Diophantine approximation, Cambridge Tracts in Mathematics and Mathematical Physics, No. 45, Cambridge Univ. Press, 1957.

[6] L. H. Eliasson - «Perturbations of stable invariant tori for Hamiltonian systems », Ann. Scuola Norm. Sup. Pisa Cl. Sci. 15 (1988), p. 115-147.

[7] R. S. Hamilton - «The inverse function theorem of Nash and Moser», Bull. Amer. Math. Soc. (N.S.) 7 (1982), p. 65-222.

[8] M.-R. HERMAN - «Sur la conjugaison différentiable des difféomorphismes du cercle à des rotations », Publ. Math. I.H.É.S. 49 (1979), p. 5-233.

[9] _ « Sur les courbes invariantes par les difféomorphismes de l'anneau. Vol. 1 », Astérisque 103 (1983).

[10] _ « Une méthode pour minorer les exposants de Lyapounov et quelques exemples montrant le caractère local d'un théorème d'Arnol'd et de Moser sur le tore de dimension 2 », Comment. Math. Helv. 58 (1983), p. $453-502$.

[11] « «imple proofs of local conjugacy theorems for diffeomorphisms of the circle with almost every rotation number », Bol. Soc. Brasil. Mat. 16 (1985), p. 45-83.

[12] L. HöRMANDER - « The boundary problems of physical geodesy », Arch. Rational Mech. Anal. 62 (1976), p. 1-52.

[13] T. H. JÄGER \& G. KELLER - «The Denjoy type of argument for quasiperiodically forced circle diffeomorphisms », Ergodic Theory Dynam. Systems 26 (2006), p. 447-465.

[14] R. Johnson \& J. Moser - « The rotation number for almost periodic potentials », Comm. Math. Phys. 84 (1982), p. 403-438.

[15] M. Jonsson - «Dynamics of polynomial skew products on $\mathbf{C}^{2} »$, Math. Ann. 314 (1999), p. 403-447. 
[16] _ « Ergodic properties of fibered rational maps », Ark. Mat. 38 (2000), p. 281-317.

[17] A. KATOK \& B. HAsselblatT - Introduction to the modern theory of dynamical systems, Encyclopedia of Mathematics and its Applications, vol. 54, Cambridge Univ. Press, 1995.

[18] Y. Katznelson - An introduction to harmonic analysis, $3^{\mathrm{e}}$ éd., Cambridge Mathematical Library, Cambridge Univ. Press, 2004.

[19] R. KRIKORIAN - «Réductibilité des systèmes produits-croisés à valeurs dans des groupes compacts », Astérisque 259 (1999).

[20] S. LANG - Introduction to diophantine approximations, Addison-Wesley Publishing Co., Reading, Mass.-London-Don Mills, Ont., 1966.

[21] E. L. Lima - Curso de análise, vol. 2, Projeto Euclides. IMPA, CNPq, 1981.

[22] V. K. MEL'NIKOV - «On certain cases of conservation of almost periodic motions with a small change of the Hamiltonian function », Dokl. Akad. Nauk SSSR 165 (1965), p. 1245-1248.

[23] M. PONCE - «Local dynamics for fibred holomorphic transformations », Nonlinearity 20 (2007), p. 2939-2955.

[24] _ « «n the persistence of invariant curves for fibered holomorphic transformations », 2009.

[25] W. M. SchmidT - Diophantine approximation, Lecture Notes in Math., vol. 785, Springer, 1980.

[26] F. Sergeraert - «Un théorème de fonctions implicites sur certains espaces de Fréchet et quelques applications », Ann. Sci. École Norm. Sup. 5 (1972), p. 599-660.

[27] O. SESTER - «Étude dynamique des polynômes fibrés », Thèse, Université de Paris-Sud, 1997.

[28] _ « Hyperbolicité des polynômes fibrés », Bull. Soc. Math. France 127 (1999), p. 393-428.

[29] J. STARK - «Transitive sets for quasi-periodically forced monotone maps », Dyn. Syst. 18 (2003), p. 351-364.

[30] M. Viana - «Multidimensional nonhyperbolic attractors », Publ. Math. I.H.É.S. 85 (1997), p. 63-96.

[31] J.-C. Yoccoz - «Théorème de Siegel, nombres de Bruno et polynômes quadratiques », Astérisque 231 (1995), p. 3-88.

[32] _ _ Analytic linearization of circle diffeomorphisms », in Dynamical systems and small divisors (Cetraro, 1998), Lecture Notes in Math., vol. 1784, Springer, 2002, p. 125-173. 